\title{
COSEBls: Extracting the full E-/B-mode information from cosmic shear correlation functions
}

\author{
P. Schneider ${ }^{1}$, T. Eifler ${ }^{2,1}$, and E. Krause ${ }^{3}$ \\ 1 Argelander-Institut für Astronomie, Universität Bonn, Auf dem Hügel 71, 53121 Bonn, Germany \\ e-mail: peter@astro.uni-bonn.de \\ 2 Center for Cosmology and Astro-Particle Physics, The Ohio State University, 191 W. Woodruff Ave., Columbus, OH 43210, USA \\ e-mail: teifler@mps.ohio-state.edu \\ 3 California Institute of Technology, Dept. of Astronomy, MC 105-24, Pasadena CA 91125, USA \\ e-mail: ekrause@astro.caltech.edu
}

Received 10 February 2010 / Accepted 29 June 2010

ABSTRACT

\begin{abstract}
Context. Cosmic shear is considered one of the most powerful methods for studying the properties of dark energy in the Universe. As a standard method, the two-point correlation functions $\xi_{ \pm}(\vartheta)$ of the cosmic shear field are used as statistical measures for the shear field.

Aims. In order to separate the observed shear into E- and B-modes, the latter being most likely produced by remaining systematics in the data set and/or intrinsic alignment effects, several statistics have been defined before. Here we aim at a complete E-/B-mode decomposition of the cosmic shear information contained in the $\xi_{ \pm}$on a finite angular interval.

Methods. We construct two sets of such E-/B-mode measures, namely Complete Orthogonal Sets of E-/B-mode Integrals (COSEBIs), characterized by weight functions between the $\xi_{ \pm}$and the COSEBIs which are polynomials in $\vartheta$ or polynomials in $\ln \vartheta$, respectively. Considering the likelihood in cosmological parameter space, constructed from the COSEBIs, we study their information content. Results. We show that the information grows with the number of COSEBI modes taken into account, and that an asymptotic limit is reached which defines the maximum available information in the E-mode component of the $\xi_{ \pm}$. We show that this limit is reached the earlier (i.e., for a smaller number of modes considered) the narrower the angular range is over which $\xi_{ \pm}$are measured, and it is reached much earlier for logarithmic weight functions. For example, for $\xi_{ \pm}$on the interval $1^{\prime} \leq \vartheta \leq 400^{\prime}$, the asymptotic limit for the parameter pair $\left(\Omega_{\mathrm{m}}, \sigma_{8}\right)$ is reached for $\sim 25$ modes in the linear case, but already for 5 modes in the logarithmic case. The COSEBIs form a natural discrete set of quantities, which we suggest as method of choice in future cosmic shear likelihood analyses.
\end{abstract}

Key words. large-scale structure of Universe - gravitational lensing: weak - cosmological parameters - methods: statistical

\section{Introduction}

The shear field in weak lensing is caused by the tidal component of the gravitational field of the mass distribution between us and a distant population of sources (see Mellier 1999; Bartelmann \& Schneider 2001; Refregier 2003; Schneider et al. 2006; Munshi et al. 2008, for recent reviews). If the shear, estimated from the image shapes of distant galaxies, is solely due to gravitational lensing, then it should consist only of a "gradient component", the so-called E-mode shear (see Crittenden et al. 2002; Schneider et al. 2002). B-modes (or curl components) cannot be generated by gravitational light deflection in leading order, and higher-order effects from lensing are expected to be small, as seen in ray-tracing simulations through the cosmological density field (e.g., Jain et al. 2000; Hilbert et al. 2009).

Therefore, the splitting of the observered shear field into its E- and B-modes is of great importance to isolate the gravitational shear from the shear components most likely not due to lensing, in order to (i) have a measure for the impact of other effects besides lensing (such as insufficient PSF correction for the shape measurements, or intrinsic alignment effects) on the observed shear field; and to (ii) isolate the lensing shear and to compare it with the expectation from cosmological models. Indeed, almost all more recent cosmic shear surveys perform such an E-/B-mode decomposition of second-order shear measures (e.g., Hoekstra et al. 2002; Jarvis et al. 2003; Hetterscheidt et al. 2007; Fu et al. 2008).
The standard technique for this separation is the aperture dispersion $\left\langle M_{\mathrm{ap}}^{2}(\theta)\right\rangle$ and $\left\langle M_{\times}^{2}(\theta)\right\rangle$ (Schneider et al. 1998), which can be calculated in terms of the shear two-point correlation functions (2PCFs) $\xi_{ \pm}(\vartheta)$ on a finite interval $0 \leq \vartheta \leq 2 \theta$. Alternatively, one can construct E- and B-mode shear correlation functions (Crittenden et al. 2002), which, however, can be calculated only if the shear correlation function $\xi_{+}$is known for arbitrarily large separations. As was pointed out by Kilbinger et al. (2006), the fact that the calculation of the aperture dispersion requires the knowledge of the shear correlation functions down to zero separation, together with the inability to measure the shape of image pairs with very small angular separation, leads to biases in the estimated values for the aperture dispersions, in particular to an effective E-/B-mode mixing.

For that reason, Schneider \& Kilbinger (2007) - hereafter SK07 - developed a new second-order shear statistics, that can be calculated from the shear correlation functions $\xi_{ \pm}$on a finite interval $\vartheta_{\min } \leq \vartheta \leq \vartheta_{\max }$ and which provides a clean separation of E- and B-modes. In particular, SK07 derived general expressions for the relation between E-/B-mode second-order shear quantities and the shear 2PCFs. They considered one particular example of such a relation, leading to the so-called the ring statistics, based solely on geometric considerations. Eifler et al. (2010) and Fu \& Kilbinger (2010) - hereafter FK10 - have shown that, although the signal-to-noise at fixed angular scale is smaller for the ring statistics than for the aperture dispersion, 
the correlation matrix between measurements at different angular scales is considerably narrower in the case of the ring statistics, yielding that the information contents of the two measures are quite comparable. Applying the ring statistics to the same cosmic shear correlation functions as used by Fu et al. (2008) in their measurement from the Canada-France-Hawaii Telescope Legacy Survey, Eifler et al. (2010) obtained a clear signal, as well as a better localization of the remaining B-modes.

In FK10, more general E-/B-mode measures have been considered, based on the general transformation derived in SK07. Specifically, FK10 have constructed E-mode quantities which maximize the signal-to-noise for a given interval $\vartheta_{\text {min }} \leq \vartheta \leq$ $\vartheta_{\max }$, or which maximize the figure of merit in parameter space, as obtained from considering the Fisher matrix. Both of the resulting E-mode statistics are by construction superior to the ring statistics, and also yield higher signal-to-noise, or a higher figure-of-merit, than the aperture dispersion.

In this paper, we construct sets of E-/B-mode measures, $E_{n}$ and $B_{n}$, based on shear correlation functions on a finite interval. In a well-defined sense, for a given angular interval $\vartheta_{\text {min }} \leq \vartheta \leq$ $\vartheta_{\text {max }}$, these second-order E-/B-mode measures form a complete set each, so that all E-B-separable information contained in the $\xi_{ \pm}(\vartheta)$ is also contained in this complete set. With these complete sets of second-order shear measures, we propose a new approach to compare observed shear correlations with model predictions. Whereas all such comparisons done hitherto define a secondorder shear measure as a function of angular scale [such as $\xi_{ \pm}(\vartheta)$ or $\left.\left\langle M_{\mathrm{ap}}^{2}(\theta)\right\rangle\right]$, the choice of the grid points in the angular scale being arbitrary, the complete set of the $E_{n}$ are a "natural" discrete set of quantities that can be used in a likelihood analysis. One can hope that a finite and possibly rather small number of the $E_{n}$ contains most of the cosmological information, depending on the choice of the set.

In Sect. 2 we summarize the general equations for E-/Bmode measures obtained from the two-point correlation functions of the shear field over a finite interval, and derive the covariance matrix for a set of such E-B-mode measures. We then construct in Sect. 3 two examples of Complete Orthogonal Sets of E-/B-mode Integrals (COSEBIs), one of them using weight functions which are polynomials in $\vartheta$, the others being polynomials in $\ln \vartheta$. In the former case, explicit relations for the corresponding weight functions are obtained for any polynomial order, whereas in the logarithmic case the coefficients have to be obtained through a matrix inversion. In Sect. 4, we then investigate the information content of these COSEBIs, by calculating the likelihood of cosmological parameter combinations and the corresponding Fisher matrix for a fiducial cosmic shear survey, using the two COSEBIs constructed, as well as the original shear correlation functions. We conclude by discussing the advantages of the COSEBIs over the other second-order shear measures that have been suggested in the literature. In Appendix B, we show how COSEBIs can be used to maximize the signal-to-noise of a cosmic shear E-mode measure. In addition we show how to construct pure E/B-mode correlation functions from the COSEBIs and relate them to the $2 \mathrm{PCF}$.

\section{E-/B-mode decomposition}

In SK07 we have shown than an E-/B-mode separation of second-order shear statistics is obtained from the $2 \mathrm{PCFs} \xi_{ \pm}$by

$E=\frac{1}{2} \int_{0}^{\infty} \mathrm{d} \vartheta \vartheta\left[T_{+}(\vartheta) \xi_{+}(\vartheta)+T_{-}(\vartheta) \xi_{-}(\vartheta)\right]$,

Page 2 of 16
$B=\frac{1}{2} \int_{0}^{\infty} \mathrm{d} \vartheta \vartheta\left[T_{+}(\vartheta) \xi_{+}(\vartheta)-T_{-}(\vartheta) \xi_{-}(\vartheta)\right]$,

provided the two weight functions $T_{ \pm}$are related through

$\int_{0}^{\infty} \mathrm{d} \vartheta \vartheta \mathrm{J}_{0}(\ell \vartheta) T_{+}(\vartheta)=\int_{0}^{\infty} \mathrm{d} \vartheta \vartheta \mathrm{J}_{4}(\ell \vartheta) T_{-}(\vartheta)$

or, equivalently,

$$
\begin{aligned}
& T_{+}(\vartheta)=T_{-}(\vartheta)+\int_{\vartheta}^{\infty} \mathrm{d} \theta \theta T_{-}(\theta)\left(\frac{4}{\theta^{2}}-\frac{12 \vartheta^{2}}{\theta^{4}}\right), \\
& T_{-}(\vartheta)=T_{+}(\vartheta)+\int_{0}^{\vartheta} \mathrm{d} \theta \theta T_{+}(\theta)\left(\frac{4}{\vartheta^{2}}-\frac{12 \theta^{2}}{\vartheta^{4}}\right) .
\end{aligned}
$$

In this case, $E$ contains only E-modes, whereas $B$ depends only on the B-mode shear. Furthermore, it was shown in SK07 that an E-mode second-order statistics is obtained from the shear correlation functions on a finite interval $\vartheta_{\min } \leq \vartheta \leq \vartheta_{\max }$ if the function $T_{+}$vanishes outside the same interval, and in addition, the two conditions

$\int_{\vartheta_{\min }}^{\vartheta_{\max }} \mathrm{d} \vartheta \vartheta T_{+}(\vartheta)=0=\int_{\vartheta_{\min }}^{\vartheta_{\max }} \mathrm{d} \vartheta \vartheta^{3} T_{+}(\vartheta)$

are satisfied; in this case, the function $T_{-}(\vartheta)$ as calculated from Eq. (3) also has finite support on the interval $\vartheta_{\text {min }} \leq \vartheta \leq \vartheta_{\text {max }}$. In SK07, a particular set of functions $T_{ \pm}$was introduced, originating from the geometrical construction of cross-correlating the shear in two non-overlapping annuli, and the corresponding estimators were termed "ring statistics".

The origin of the conditions expressed in Eq. (4) can be understood as follows: a uniform shear field cannot be assigned an E- or B-mode origin. Such a shear field gives rise to shear correlation functions of the form $\xi_{+}(\vartheta)=$ const. and $\xi_{-}(\vartheta)=0$. According to the first of Eq. (4), this component is filtered out in Eq. (1). Furthermore, one possibility to distinguish between E- and B-modes is the consideration of the vector field $\boldsymbol{u}=$ $\left(\gamma_{1,1}+\gamma_{2,2}, \gamma_{2,1}-\gamma_{1,2}\right)$ constructed from partial derivatives of the shear field $\gamma(\boldsymbol{\vartheta})$ (Kaiser 1995). A pure E-mode shear yields a vanishing curl of $\boldsymbol{u}$, whereas a pure B-mode shear leads to $\nabla \cdot \boldsymbol{u}=0$; a shear field which yields $\nabla \cdot \boldsymbol{u}=0=\operatorname{curl}(\boldsymbol{u})$ cannot be uniquely classified as E- or B-mode.

If we now consider a shear field which depends linearly on $\boldsymbol{\vartheta}$, then the vector field $\boldsymbol{u}$ is constant, and thus it cannot be uniquely split into E- and B-modes. On the other hand, such a shear field gives rise to correlation functions of the form $\xi_{+}(\vartheta)=A+B \vartheta^{2}$, $\xi_{-}(\vartheta)=0$, where $A$ and $B$ are constants. Again, the correlation function of such a shear field is filtered out due to the conditions in Eq. (4).

\subsection{E-/B-modes from a set of functions}

Of course, there are many functions $T_{+}(\vartheta)$ which satisfy the constraints in Eq. (4). Assume we construct a set of functions $T_{+n}(\vartheta)$ which all satisfy Eq. (4) and which are, in a way specified later, orthogonal. Then one can construct the corresponding $T_{-n}(\vartheta)$ from Eq. (3), and thus one obtains the set $E_{n}$ and $B_{n}$ of secondorder shear measures with a clean E-/B-mode separation. Each of the $E_{n}$ and $B_{n}$ measures an integral over the power spectrum of E- and B-modes, respectively,

$E_{n}=\int_{0}^{\infty} \frac{\mathrm{d} \ell \ell}{2 \pi} P_{\mathrm{E}}(\ell) W_{n}(\ell)$,
$B_{n}=\int_{0}^{\infty} \frac{\mathrm{d} \ell \ell}{2 \pi} P_{\mathrm{B}}(\ell) W_{n}(\ell)$, 
where the filter functions are

$W_{n}(\ell)=\int_{\vartheta_{\min }}^{\vartheta_{\max }} \mathrm{d} \vartheta \vartheta T_{+n}(\vartheta) \mathrm{J}_{0}(\ell \vartheta)$,

and where we made use of the relation between the shear correlation functions and the power spectra (see, e.g., Schneider et al. 2002)

$\xi_{+}(\vartheta)=\int_{0}^{\infty} \frac{\mathrm{d} \ell \ell}{2 \pi} \mathrm{J}_{0}(\ell \vartheta)\left[P_{\mathrm{E}}(\ell)+P_{\mathrm{B}}(\ell)\right]$,
$\xi_{-}(\vartheta)=\int_{0}^{\infty} \frac{\mathrm{d} \ell \ell}{2 \pi} \mathrm{J}_{4}(\ell \vartheta)\left[P_{\mathrm{E}}(\ell)-P_{\mathrm{B}}(\ell)\right]$.

We next calculate the covariance of the E- and B-mode measures making use of Eq. (5),

$$
\begin{aligned}
C_{m n}^{\mathrm{E}} & \equiv\left\langle E_{m} E_{n}\right\rangle-\left\langle E_{m}\right\rangle\left\langle E_{n}\right\rangle \\
& =\int_{0}^{\infty} \frac{\mathrm{d} \ell \ell}{2 \pi} W_{m}(\ell) \int_{0}^{\infty} \frac{\mathrm{d} \ell^{\prime} \ell^{\prime}}{2 \pi} W_{n}\left(\ell^{\prime}\right)\left\langle\Delta P_{\mathrm{E}}(\ell) \Delta P_{\mathrm{E}}\left(\ell^{\prime}\right)\right\rangle \\
& =\frac{1}{\pi A} \int_{0}^{\infty} \mathrm{d} \ell \ell W_{m}(\ell) W_{n}(\ell)\left[P_{\mathrm{E}}(\ell)+N_{\epsilon}\right]^{2},
\end{aligned}
$$

where in the final step we have assumed a Gaussian shear field and used the corresponding expression for the covariance of the power spectrum from Joachimi et al. (2008). Here, $A$ is the survey area, $N_{\epsilon}=\sigma_{\epsilon}^{2} /(2 \bar{n})$ is the amplitude of the white noise power spectrum resulting from the intrinsic ellipticity distribution of sources, $\sigma_{\epsilon}$ is the dispersion of the intrinsic ellipticity, and $\bar{n}$ is the mean number density of sources. The covariance of the $B_{n}$, $C_{m n}^{\mathrm{B}}$, has exactly the same form, with $P_{\mathrm{E}}$ replaced by $P_{\mathrm{B}}$, and the covariance between the $E_{n}$ and $B_{m}$ vanishes.

As a consistency check, we calculate the covariance in a different form, starting from the relation between the $E_{n}$ and the shear correlation functions. We then obtain

$$
\begin{aligned}
C_{m n}^{\mathrm{E}}= & \frac{1}{4} \int_{\vartheta_{\min }}^{\vartheta_{\max }} \mathrm{d} \vartheta \vartheta \int_{\vartheta_{\min }}^{\vartheta_{\max }} \mathrm{d} \vartheta^{\prime} \vartheta^{\prime} \\
& \times \sum_{\mu, \nu=\{+,-\}} T_{\mu \mathrm{m}}(\vartheta) T_{\nu n}\left(\vartheta^{\prime}\right) C_{\mu \nu}\left(\vartheta, \vartheta^{\prime}\right),
\end{aligned}
$$

where $C_{ \pm \pm}\left(\vartheta, \vartheta^{\prime}\right)$ is the covariance of the shear correlation function $\xi_{ \pm}(\vartheta)$. Using the relations of Joachimi et al. (2008) for the covariance of the $\xi_{ \pm}$, assuming a Gaussian shear field, and making use of Eq. (2), the result (8) is re-obtained.

The comparison of the $E_{n}^{\text {obs }}$ obtained from observations with those of a model $E_{n}(\pi)$, where $\pi$ denotes a set of $M$ model parameters, can then be done via

$\chi^{2}=\sum_{m, n=1}^{N}\left[E_{m}^{\mathrm{obs}}-E_{m}(\pi)\right]\left(C^{\mathrm{E}}\right)_{m n}^{-1}\left[E_{n}^{\mathrm{obs}}-E_{n}(\pi)\right]$,

where $N$ is the maximum number of E-modes considered, or with a likelihood function

$\mathcal{L}=\left[(2 \pi)^{N / 2} \sqrt{\operatorname{det} C^{\mathrm{E}}}\right]^{-1} \mathrm{e}^{-\chi^{2} / 2}$.

\subsection{Calculation of E-mode second-order statistics from ray-tracing simulations}

Due to the limited range of validity of analytic approximations for the calculation of cosmic shear statistics, ray tracing through $N$-body simulated three-dimensional density distributions are carried out (see, e.g., Jain et al. 2000; Hilbert et al. 2009, and references therein). As shown in these papers, the resulting B-mode shear is several orders of magnitude smaller than the E-mode shear, so that the resulting shear field can be described very accurately in terms of an equivalent surface mass density $\kappa(\boldsymbol{\theta})$. It is often faster to derive statistical properties of the resulting shear field from the corresponding properties of the $\kappa$-field. For example, the aperture mass $M_{\text {ap }}$ (Schneider 1996) can be obtained from the shear field through a radial filter function $Q$, but also from the $\kappa$-field through a related radial filter function $U$. Hence, one can calculate the field of $M_{\text {ap }}$ from the equivalent surface mass density, convolved with the filter $U$, and the aperture mass dispersion is then given as the dispersion of this field. In this way, no correlation functions of the shear need to be obtained for making predictions, saving computation time.

Here we will show that, similar to the case of the aperture mass dispersion, the E-mode second-order shear statistics defined in Eq. (1) can be obtained from a simulated $\kappa$-field, without the need to calculate the shear correlation functions. For that we note that, in the absence of B-modes, one has

$E=\int_{0}^{\infty} \mathrm{d} \vartheta \vartheta T_{+}(\vartheta) \xi_{+}(\vartheta)$

and that the correlation functions of $\kappa$ and $\gamma$ agree,

$\left\langle\kappa(\boldsymbol{\theta}) \kappa\left(\boldsymbol{\theta}^{\prime}\right)\right\rangle=\left\langle\gamma(\boldsymbol{\theta}) \gamma^{*}\left(\boldsymbol{\theta}^{\prime}\right)\right\rangle=\xi_{+}\left(\left|\boldsymbol{\theta}-\boldsymbol{\theta}^{\prime}\right|\right)$.

If we smooth the convergence field with a radial filter function $F$, obtaining

$\kappa_{\mathrm{s}}(\boldsymbol{\theta})=\int \mathrm{d}^{2} \theta^{\prime} \kappa\left(\boldsymbol{\theta}^{\prime}\right) F\left(\left|\boldsymbol{\theta}-\boldsymbol{\theta}^{\prime}\right|\right)$,

the correlator of the smoothed field with the unsmoothed field at zero lag becomes

$\left\langle\kappa(\boldsymbol{\theta}) \kappa_{\mathrm{S}}(\boldsymbol{\theta})\right\rangle=\int \mathrm{d}^{2} \theta^{\prime} F\left(\left|\boldsymbol{\theta}-\boldsymbol{\theta}^{\prime}\right|\right) \xi_{+}\left(\left|\boldsymbol{\theta}-\boldsymbol{\theta}^{\prime}\right|\right)$.

Setting $\boldsymbol{\vartheta}=\boldsymbol{\theta}^{\prime}-\boldsymbol{\theta}$, we see that

$E=\left\langle\kappa(\boldsymbol{\theta}) \kappa_{\mathrm{s}}(\boldsymbol{\theta})\right\rangle$,

if we choose $F(\vartheta)=(2 \pi)^{-1} T_{+}(\vartheta)$. Hence, the calculation of $E$ from simulations can proceed by convolving the $\kappa$-field with the function $T_{+}(\vartheta) /(2 \pi)$, and correlating the resulting field with the original $\kappa$-field, dropping a band of width $\vartheta_{\text {max }}$ along the boundaries of the field where the convolution via FFT causes artifacts.

\section{Complete sets of weight functions}

Here, we construct complete sets of functions which satisfy the constraints (4) for the weight function $T_{+}(\vartheta)$ on the interval $\vartheta_{\min } \leq \vartheta \leq \vartheta_{\max }$. It should be noted that, once a complete set of such functions is known, the maximization of the signal-tonoise of the second-order E-mode shear - a problem considered in FK10 - reduces to a linear algebra problem, as shown in Appendix B.

Readers less interested in the explicit construction of these COSEBIs can go directly to Sect. 4. 

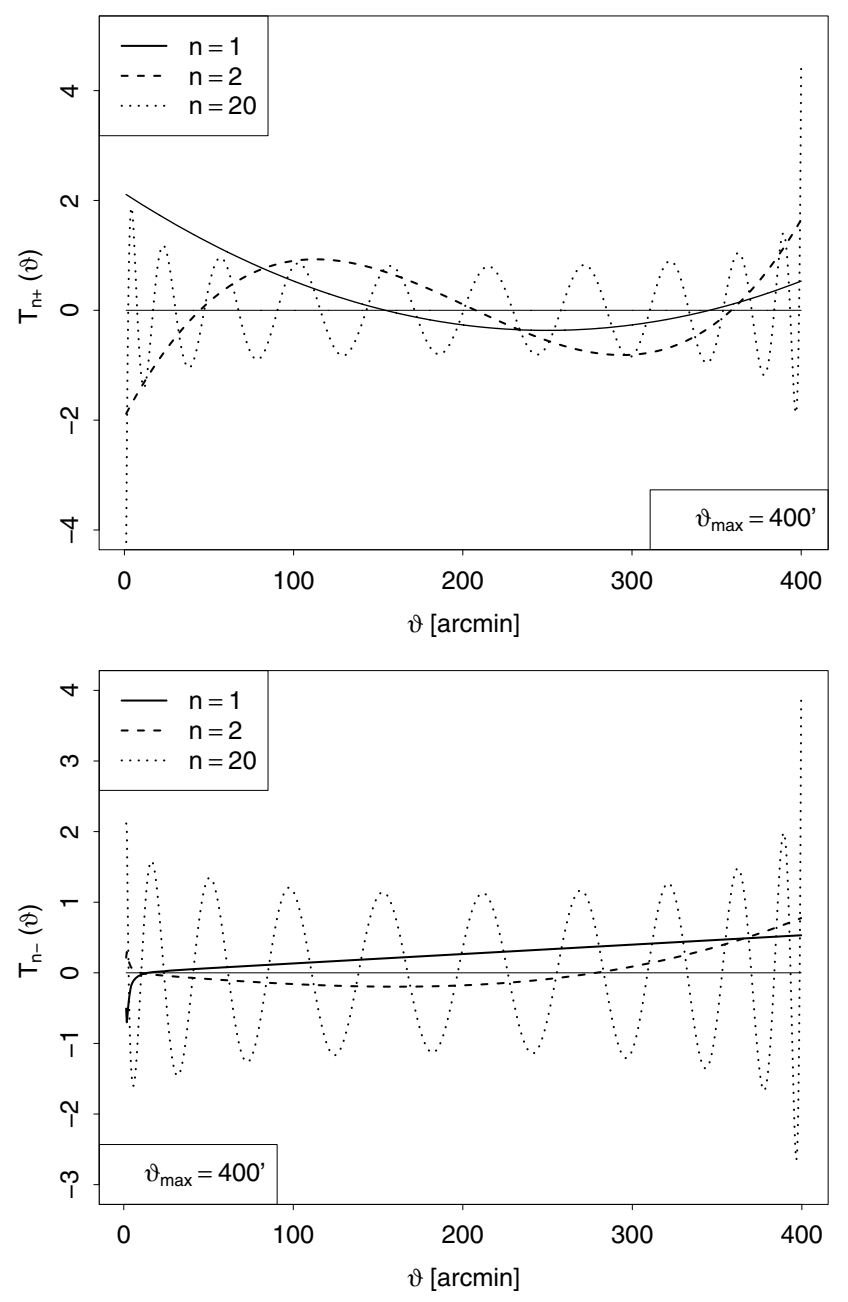

Fig. 1. The linear filter functions $T_{ \pm n}(\vartheta)$ for $\vartheta_{\min }=1^{\prime}, \vartheta_{\max }=400^{\prime}$. Note that the shape of the curves depends only on the ratio $\vartheta_{\min } / \vartheta_{\max }$.

\subsection{Polynomial weight functions}

First, we construct a complete set of weight functions which are polynomials in $\vartheta$. To do so, we transform the interval $\vartheta_{\min } \leq \vartheta \leq$ $\vartheta_{\max }$ onto the unit interval $-1 \leq x \leq 1$, by defining

$x=\frac{2(\vartheta-\bar{\vartheta})}{\Delta \vartheta}$

with $\bar{\vartheta}=\left(\vartheta_{\min }+\vartheta_{\max }\right) / 2, \Delta \vartheta=\vartheta_{\max }-\vartheta_{\min }$. In addition, we define the relative interval width $B=\Delta \vartheta /(2 \bar{\vartheta})=\left(\vartheta_{\max }-\right.$ $\left.\vartheta_{\text {min }}\right) /\left(\vartheta_{\text {max }}+\vartheta_{\text {min }}\right)$. Thus, as $\vartheta$ varies from $\vartheta_{\text {min }}$ to $\vartheta_{\text {max }}, x$ goes from -1 to +1 . Then we set $T_{+n}(\vartheta)=t_{+n}(x)$, and $T_{-n}(\vartheta)=t_{-n}(x)$. The $t_{+n}$ are chosen to be polynomials in $x$; as Eq. (15) is a linear transformation, the polynomial order is preserved. Furthermore, we require that the set of functions are orthonormal, i.e.,

$$
\int_{-1}^{1} \mathrm{~d} x t_{+n}(x) t_{+m}(x)=\delta_{m n}
$$

The first two functions of the set are constructed "by hand": the lowest-order polynomial which can satisfy the constraints (4) and the normalization constraint (16) is of second order. Hence, we choose $t_{+1}(x)$ to be a second-order polynomial, and determine its three coefficients from the three constraints. The lowest-order polynomial which can satisfy the two constraints (4) and the orthonormality relation (16) for $m=1,2$ is of third order, and its four coefficients are determined accordingly; this yields

$$
\begin{aligned}
t_{+1}(x)= & \frac{1}{\sqrt{X_{1}}}\left[3 B^{2}-5-6 B x+3\left(5-B^{2}\right) x^{2}\right] \\
t_{+2}(x)= & \frac{1}{\sqrt{X_{2}}}\left[B^{3}\left(25+3 B^{2}\right)-15\left(35+9 B^{2}+8 B^{4}\right) x\right. \\
& \left.-15 B^{3}\left(3+B^{2}\right) x^{2}+35\left(25+5 B^{2}+6 B^{4}\right) x^{3}\right]
\end{aligned}
$$

with

$$
\begin{aligned}
& X_{1}=8\left(25+5 B^{2}+6 B^{4}\right) / 5, \\
& X_{2}=8\left(25+5 B^{2}+6 B^{4}\right)\left(175+35 B^{2}+45 B^{4}+B^{6}\right) .
\end{aligned}
$$

To obtain the higher-order functions of this set, we note that the Legendre polynomials $P_{n}(x)$ are orthogonal, and that

$\int_{-1}^{1} \mathrm{~d} x P_{n}(x) x^{m}=0$ for $m<n$.

This shows that the constraints (4), written in terms of $x$, are satisfied if we choose $t_{+}(x) \propto P_{n}(x)$ for all $n \geq 4$. Furthermore, the $P_{n}(x)$ for $n \geq 4$ are orthogonal to $t_{+1}(x)$ and $t_{+2}(x)$, since the latter are polynomials of order $\leq 3$. Thus, choosing the normalization such as to satisfy Eq. (16), we find for $n \geq 3$,

$t_{+n}(x)=\sqrt{\frac{2 n+3}{2}} P_{n+1}(x) \equiv p_{n+1}(x)$.

In the upper panel of Fig. 1, we have plotted the filter function $T_{+n}(\vartheta)$ for three values of $n$. For $n \geq 3$, they are simply proportional to the Legendre polynomials. Note that $T_{+n}(\vartheta)$ has $(n+1)$ roots in the interval $\vartheta_{\text {min }} \leq \vartheta \leq \vartheta_{\text {max }}$, and the normalization is chosen such that $T_{+n}\left(\vartheta_{\max }\right)>0$. The corresponding filter functions $W_{n}(\ell)$ which relate the COSEBIs to the power spectrum $P_{\mathrm{E}}(\ell)$ are displayed in Fig. 2, for several values of $n$ and for two different values of the relative width parameter $B$ (corresponding to two different values of $\vartheta_{\max }$ ).

For this set of functions $t_{+n}(x)$, we can obtain the corresponding $t_{-n}(x)$ using Eq. (3),

$t_{-}(x)=t_{+}(x)+\frac{4 B}{(1+B x)^{2}} \int_{-1}^{x} \mathrm{~d} y t_{+}(y) G(y, x)$,

where

$G(y, x)=1+B y-3 \frac{(1+B y)^{3}}{(1+B x)^{2}}=\sum_{k=0}^{3} A_{k} y^{k}$,

and the coefficients $A_{k}$ are given explicitly as

$A_{0}=1-\frac{3}{(1+B x)^{2}}, \quad A_{1}=B-\frac{9 B}{(1+B x)^{2}}$,

$A_{2}=\frac{-9 B^{2}}{(1+B x)^{2}}, \quad A_{3}=\frac{-3 B^{3}}{(1+B x)^{2}}$.

For the first two functions, the integral is carried out explicitly, yielding

$t_{-1}(x)=\frac{1}{\sqrt{X_{1}}(1+B x)^{4}} \sum_{k=0}^{5} U_{1 k} x^{k}$
$t_{-2}(x)=\frac{1}{\sqrt{X_{2}}(1+B x)^{4}} \sum_{k=0}^{7} U_{2 k} x^{k}$ 
P. Schneider et al.: COSEBIs: Extracting the full E-/B-mode information from cosmic shear correlation functions
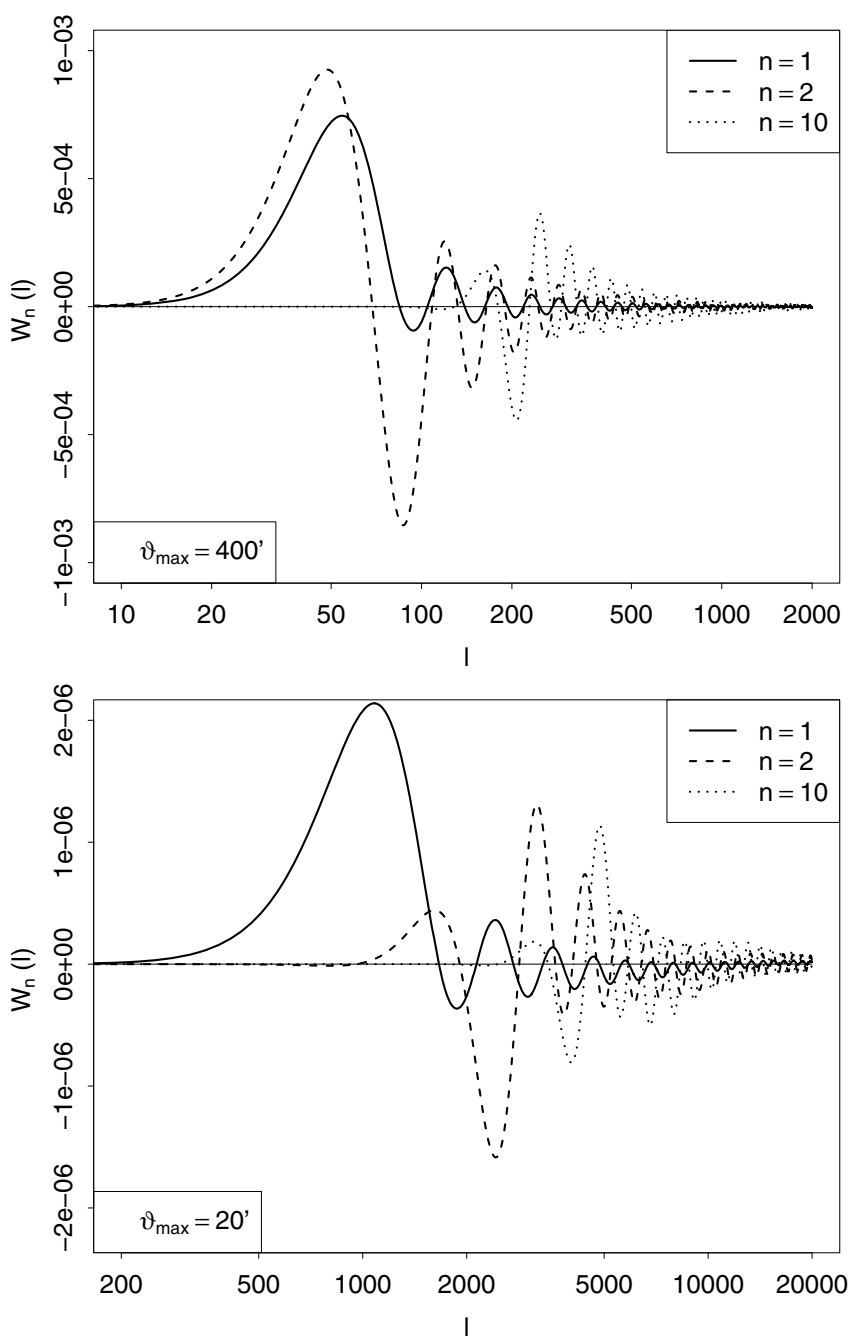

Fig. 2. The functions $W_{n}$ as defined in Eq. (6) which relate the COSEBIs to the underlying power spectrum, calculated from the $T_{ \pm n}$. The upper panel corresponds to $\vartheta_{\max }=400^{\prime}$, whereas the lower panel is calculated using $\vartheta_{\max }=20^{\prime}$, both for $\vartheta_{\min }=1^{\prime}$.

with the coefficients $U$

$$
\begin{aligned}
& U_{10}=-5+19 B^{2}-15 B^{4}+3 B^{6}, \\
& U_{11}=2 B\left(7+B^{2}-3 B^{4}\right), \\
& U_{12}=15+7 B^{2}+B^{4}-3 B^{6}, \\
& U_{13}=20 B, \quad U_{14}=10 B^{2}, \quad U_{15}=2 B^{3} ; \\
& U_{20}=-B\left(350-360 B^{2}+182 B^{4}-93 B^{6}+21 B^{8}\right), \\
& U_{21}=-525+215 B^{2}-30 B^{4}+38 B^{6}+18 B^{8}, \\
& U_{22}=B^{3}\left(130+30 B^{2}+19 B^{4}+9 B^{6}\right), \\
& U_{23}=5\left(175+105 B^{2}+48 B^{4}+12 B^{6}\right), \\
& U_{24}=5 B\left(350+105 B^{2}+87 B^{4}+6 B^{6}\right), \\
& U_{25}=B^{2}\left(1400+315 B^{2}+339 B^{4}+6 B^{6}\right), \\
& U_{26}=21 B^{3}\left(25+5 B^{2}+6 B^{4}\right), \\
& U_{27}=3 B^{4}\left(25+5 B^{2}+6 B^{4}\right) .
\end{aligned}
$$

For $n \geq 3$, we first define

$I_{n}^{k}(x):=\int_{-1}^{x} \mathrm{~d} y P_{n}(y) y^{k}$.
For $k=0$, one obtains

$$
I_{n}^{0}(x)=\frac{P_{n+1}(x)-P_{n-1}(x)}{1+2 n},
$$

whereas for $k \geq 1$, we make use of the recurrence relation for Legendre polynomials, $(2 n+1) y P_{n}(y)=(n+1) P_{n+1}(y)+$ $n P_{n-1}(y)$, to find

$I_{n}^{k}(x)=\frac{(n+1) I_{n+1}^{k-1}(x)+n I_{n-1}^{k-1}(x)}{2 n+1}$.

Making use of Eqs. (20) and (21), we then find, for $n \geq 3$,

$t_{-n}(x)=t_{+n}(x)+\sqrt{\frac{2 n+3}{2}} \frac{4 B}{(1+B x)^{2}} \sum_{k=0}^{3} A_{k} I_{n+1}^{k}(x)$.

For three different values of $n$ and $\vartheta_{\min }=1^{\prime}, \vartheta_{\max }=400^{\prime}$, the functions $T_{-n}(\vartheta)$ are displayed in the lower panel of Fig. 1.

\subsection{Logarithmic weight functions}

Choosing the $T_{+n}$ to be polynomials in $\vartheta$ implies that the structure of these weight functions is similar on all angular scales from $\vartheta_{\min }$ to $\vartheta_{\max }$. For example, the roots of the $T_{+n}$ are fairly evenly spread on the interval $\vartheta_{\text {min }} \leq \vartheta \leq \vartheta_{\text {max }}$. On the other hand, we expect the correlation function $\xi_{+}(\vartheta)$ to show more structure on small scales than on large scales. Hence, for a given maximum number $N$ of modes, the large angular scales will be sampled on finer scales than needed, whereas small angular scales may not be sufficiently well resolved to extract all information contained in the correlation function.

In order obtain a finer sampling of the small-scale correlation function for a given $N$, we now construct a set of weight functions which are polynomials in $\ln \vartheta$. Hence the roots of these weight functions are approximately evenly spaced in $\ln \vartheta$, thus the weight functions sample small angular scales with higher resolution than large angular scales. As in Sect. 3.1, this set of functions must fulfill the constraints (4), and we require the functions to be orthonormal. Hence, the lowest-order weight function again is of second-order. We parametrize this set of weight functions as

$t_{+n}^{\log }(z)=\sum_{j=0}^{n+1} c_{n j} z^{j}=N_{n} \sum_{j=0}^{n+1} \bar{c}_{n j} z^{j}$

where we choose

$z=\ln \left(\vartheta / \vartheta_{\min }\right)$,

which varies from 0 to $z_{\max }=\ln \left(\vartheta_{\max } / \vartheta_{\min }\right)$ as $\vartheta$ goes from $\vartheta_{\text {min }}$ to $\vartheta_{\text {max }}$. Furthermore, we defined $c_{n j}=N_{n} \bar{c}_{n j}$ with $N_{n} \equiv$ $c_{n(n+1)} \neq 0$, so that $\bar{c}_{n(n+1)}=1$. In this way, the relative amplitude of the $c$ 's is decoupled from the overall normalization $N_{n}$. As before, we set $T_{+n}^{\log }(\vartheta)=t_{+n}^{\log }(z)$ and $T_{-n}^{\log }(\vartheta)=t_{-n}^{\log }(z)$. With this transformation of variables the constraints (4) become

$\int_{0}^{z_{\max }} \mathrm{d} z \mathrm{e}^{2 z} t_{+n}^{\log }(z)=0=\int_{0}^{z_{\max }} \mathrm{d} z \mathrm{e}^{4 z} t_{+n}^{\log }(z)$,

and an orthonormality condition analogous to Eq. (16) can be written as

$$
\begin{aligned}
\frac{1}{\Delta \vartheta} \int_{\vartheta_{\min }}^{\vartheta_{\max }} \mathrm{d} \vartheta T_{+n}^{\log }(\vartheta) T_{+m}^{\log }(\vartheta)= \\
\\
\frac{\vartheta_{\min }}{\Delta \vartheta} \int_{0}^{z_{\max }} \mathrm{d} z \mathrm{e}^{z} t_{+n}^{\log }(z) t_{+m}^{\log }(z)=\delta_{\mathrm{nm}} .
\end{aligned}
$$


$\operatorname{Nmax}=20 ; \operatorname{tmin}=1 ; \operatorname{tmax}=400 ; \mathrm{zm}=\log [$ Rationalize[tmax/tmin] $]$

gamm $\left[\mathrm{a}_{-}, \mathrm{z}_{-}\right]=$Gamma $[\mathrm{a}, \boldsymbol{0}, \mathrm{z}]$

Do []$[k, j]=\operatorname{Re}\left[N\left[\right.\right.$ gamm $\left.\left.\left.[j+1,-k \quad z m] /(-k)^{\wedge}(j+1), 130\right]\right],\{k, 1,2\},\{j, 0,2 \quad N m a x+1\}\right]$

Do []$\left.[4, j]=\operatorname{Re}\left[N\left[\operatorname{gamm}[j+1,-4 \mathrm{zm}] /(-4)^{\wedge}(j+1), 130\right]\right],\{j, \mathbb{0}, 2 \quad \mathrm{Nmax}+1\}\right]$

Do[

Do $[a[n, j]=][2, j] /][2, n+1] ; a[n+1, j]=][4, j] /][4, n+1],\{j, 0, n\}] ; b[n]=-1 ; b[n+1]=-1 ;$

Do $[a[m, j]=N S u m[][1, i+j] \quad c[m, i],\{i, \mathbb{Q}, m+1\}$, WorkingPrecision->80, NSumTerms $->$ Nmax $],\{m, 1, n-1\},\{j, \mathbb{Q}, n\}]$;

Do $[b b[m]=-N S u m[][1, i+n+1] c[m, i],\{i, 0, m+1\}$, WorkingPrecision- $>80$, NSumTerms- $>$ Nmax $],\{m, 1, n-1\}]$;

Do $[a[m, j]=a[m, j] / b b[m],\{m, 1, n-1\},\{j, \theta, n\}] ; \operatorname{Do}[b[m]=1,\{m, 1, n-1\}]$;

$\mathrm{A}=$ Table $[a[i, j],\{\mathrm{i}, 1, \mathrm{n}+1\},\{\mathrm{j}, 0, \mathrm{n}\}] ; \mathrm{B}=\operatorname{Table}[\mathrm{b}[\mathrm{i}],\{\mathrm{i}, 1, \mathrm{n}+1\}]$;

CC=LinearSolve $[A, B] ; \operatorname{Do}[c[n, j]=C C[[j+1]],\{j, \mathbb{Q}, n\}] ; c[n, n+1]=1$;

tt $\left[n, z_{-}\right]=$Simplify $\left[\operatorname{Sum}\left[c[n, j] \quad z^{\wedge} j,\{j, 0, n+1\}\right]\right]$;

roots $=N$ Solve $[\operatorname{tt}[n, z]==0, z] ; \operatorname{Do}[r[n, j]=N[\operatorname{roots}[[j, 1,2]], 8],\{j, 1, n+1\}]$;

$\mathrm{t}\left[\mathrm{n}, \mathrm{z}_{-}\right]=\operatorname{Product}[(\mathrm{z}-\mathrm{r}[\mathrm{n}, \mathrm{j}]),\{j, 1, \mathrm{n}+1\}]$;

normgral=NIntegrate $\left[\operatorname{Exp}[\mathrm{z}] \mathrm{t}[\mathrm{n}, \mathrm{z}]^{\wedge} 2,\{\mathrm{z}, \mathbb{0}, \mathrm{zm}\}\right.$, WorkingPrecision- $\left.>50\right]$;

$\operatorname{norm}[\mathrm{n}]=\operatorname{Sqrt}[(\operatorname{Exp}[\mathrm{zm}]-1) /$ normgral $] ; \mathrm{t}\left[\mathrm{n}, \mathrm{z}_{-}\right]=\mathrm{t}[\mathrm{n}, \mathrm{z}]$ norm $[\mathrm{n}]$,

$\{\mathrm{n}, 1, \mathrm{Nmax}\}]$

ROOTS=Table $[r[n, j],\{n, 1, \operatorname{Nmax}\},\{j, 1, N \max +1\}]$

Fig. 3. Mathematica (Wolfram 1991) program to calculate the roots in Eq. (36) - they are stored with 8 significant digits in the lower left halve of the table R0OTS. Furthermore, the array norm[n] contains the normalization coefficients $N_{n}$.

To write these constraints in a more compact form we define the set of coefficients

$J(k, j)=\int_{0}^{z_{\max }} \mathrm{d} z \mathrm{e}^{k z} z^{j}=\frac{\gamma\left(j+1,-k z_{\max }\right)}{(-k)^{j+1}}$,

where $\gamma(a, x)$ is the incomplete Gamma function.

With the representation (28), the constraints (30) become

$\sum_{j=0}^{n} \bar{c}_{n j} J(2, j)=-J(2, n+1)$,
$\sum_{j=0}^{n} \bar{c}_{n j} J(4, j)=-J(4, n+1)$.

These two equations determine the two coefficients $\bar{c}_{10}, \bar{c}_{11}$ needed to obtain $t_{+1}^{\log }(z)$. We then obtain the corresponding coefficients $\bar{c}_{n j}$ by iterating in $n$. Thus, for a given $n$, we assume that the $\bar{c}_{m j}$ have been determined for all $m<n$. Then, the $\bar{c}_{n j}$ are obtained from the two Eqs. (33), and the $(n-1)$ orthogonality conditions (31) for $1 \leq m \leq n-1$, which read in the representation (28)

$\sum_{j=0}^{n+1} \sum_{i=0}^{m+1} J(1, i+j) \bar{c}_{m i} \bar{c}_{n j}=0$

or

$\sum_{j=0}^{n}\left(\sum_{i=0}^{m+1} J(1, i+j) \bar{c}_{m i}\right) \bar{c}_{n j}=-\sum_{i=0}^{m+1} J(1, i+n+1) \bar{c}_{m i}$,

where we used that $\bar{c}_{n(n+1)}=1$. Thus, together we have $n+1$ linear equations for the $n+1$ unknown coefficients $\bar{c}_{n j}, 0 \leq j \leq n$, which in principle can be readily solved (but see below). Finally, to obtain the normalization of the functions, we use Eq. (31) for $m=n$, which together with Eq. (28) yields

$N_{n}^{2} \sum_{i, j=0}^{n+1} \bar{c}_{n i} \bar{c}_{n j} J(1, i+j)=\frac{\Delta \vartheta}{\vartheta_{\min }}=\mathrm{e}^{z_{\max }}-1$,

which determines $N_{n}$ (and thus the $c_{n j}=N_{n} \bar{c}_{n j}$ ) up to an (arbitrary) sign. For definiteness, we choose the sign such that $t_{+n}^{\log }\left(z_{\max }\right)>0$, implying that $N_{n}=c_{n(n+1)}>0$.
It turns out that the solution of the system of linear equations for the c's requires very high numerical accuracy for even moderately large $n$, in particular for large values of $\vartheta_{\max } / \vartheta_{\text {min }}$. We used Mathematica (Wolfram 1991) with large setting of WorkingPrecision for calculating the incomplete Gamma function and for carrying out the sums in Eq. (34). Once the $c$ 's have been determined, the integrals in Eqs. (30) and (31) the latter for $m<n$ - have been calculated to check the accuracy of the solution. We found that, for $\vartheta_{\max } / \vartheta_{\text {min }}=400$ and for $n_{\max }=20$, one needs to determine the $c$ 's to 40 significant digits, in order for all these integrals, which should be zero, to attain values less than 0.1 . We then calculated the $n+1$ roots $r_{n, i}$ of the $t_{+n}^{\log }(z)$, and represented the functions as

$t_{+n}^{\log }(z)=N_{n} \prod_{i=1}^{n+1}\left(z-r_{n i}\right)$

For the same parameters as before, using only five significant digits for the $r$ 's renders all the integrals zero to better than $10^{-6}$, and with eight significant digits, the integrals are zero to better than $10^{-17}$ even for $n_{\max }=40$. Thus, the representation (36) is the adequate one for practical work. A short Mathematica program for calculating the $r_{n}$ is displayed in Fig. 3 .

The corresponding $T_{-n}^{\log }$ are constructed from Eq. (3), by defining $y=\ln \left(\theta / \vartheta_{\min }\right)$, which yields

$$
\begin{aligned}
t_{-n}^{\log }(z)= & t_{+n}^{\log }(z)+4 \int_{0}^{z} \mathrm{~d} y t_{+n}^{\log }(y)\left(\mathrm{e}^{2(y-z)}-3 \mathrm{e}^{4(y-z)}\right) \\
= & t_{+n}^{\log }(z) 4 \sum_{j=0}^{n+1} c_{n j} \int_{0}^{z} \mathrm{~d} y y^{j}\left(\mathrm{e}^{2(y-z)}-3 \mathrm{e}^{4(y-z)}\right) \\
= & t_{+n}^{\log }(z) 4 \mathrm{e}^{-2 z} \sum_{j=0}^{n+1} \frac{c_{n j}}{(-2)^{j+1}}[\gamma(j+1,-2 z) \\
& \left.-\frac{3 \mathrm{e}^{-2 z}}{2^{j+1}} \gamma(j+1,-4 z)\right]
\end{aligned}
$$

Given the remarks above, the first of these expressions (i.e., numerical integration) is the method of choice if the $t_{+n}^{\log }(z)$ are 
P. Schneider et al.: COSEBIs: Extracting the full E-/B-mode information from cosmic shear correlation functions
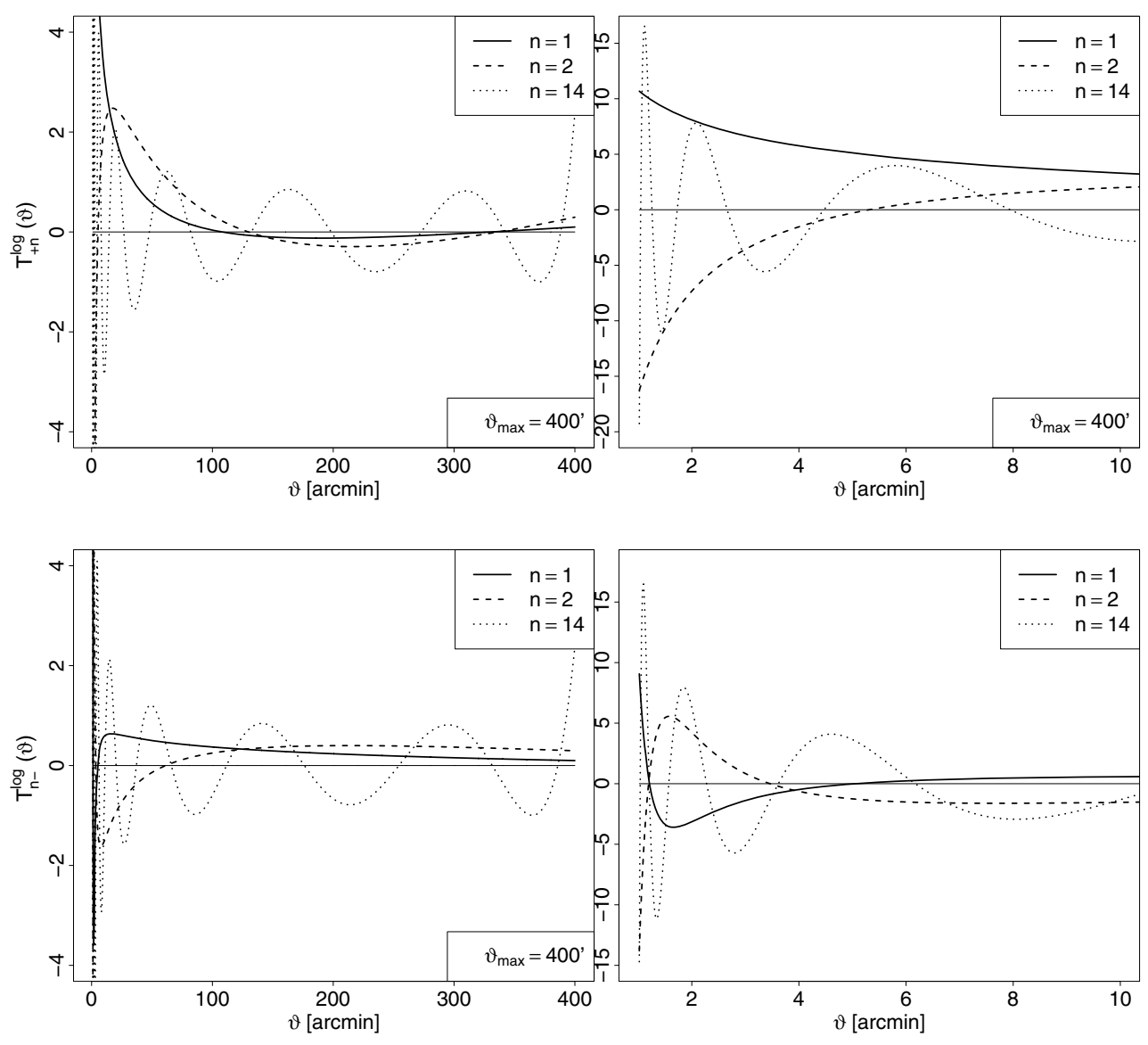

Fig. 4. The logarithmic filter functions $T_{+n}^{\log }$ for $\vartheta_{\min }=1^{\prime}$ and $\vartheta_{\max }=400^{\prime}$. The left panel shows the function over the whole interval, whereas the right panel provides a more detailed view for small $\vartheta$.

Fig. 5. The logarithmic filter functions $T_{-n}^{\log }$ for $\vartheta_{\min }=1^{\prime}$ and $\vartheta_{\max }=400^{\prime}$. As in Fig. 4 , the left panel shows the function over the whole interval, whereas the right panel provides a more detailed view for small $\vartheta$. given in the form (36). Alternatively, making use of the representation

$\gamma(j+1, z)=j !\left[1-\mathrm{e}^{-z} \sum_{m=0}^{j} \frac{z^{m}}{m !}\right]$,

one can write the $t_{-n}^{\log }(z)$ as

$t_{-n}^{\log }(z)=a_{n 2} \mathrm{e}^{-2 z}-a_{n 4} \mathrm{e}^{-4 z}+\sum_{m=0}^{n} d_{n m} z^{m}$,

where the coefficients are given as

$a_{n 2}=4 \sum_{j=0}^{n+1} \frac{c_{n j} j !}{(-2)^{j+1}}, \quad a_{n 4}=12 \sum_{j=0}^{n+1} \frac{c_{n j} j !}{(-4)^{j+1}}$,

$d_{n m}=c_{n m}+\frac{4}{m !} \sum_{j=m}^{n+1} c_{n j} j !(-2)^{m-j-1}\left(32^{m-j-1}-1\right)$.

In Figs. 4 and 5, we have plotted the filter functions $T_{ \pm n}^{\log }$ for $\vartheta_{\text {min }}=1^{\prime}$ and $\vartheta_{\text {max }}=400^{\prime}$. The left panels show these filter functions over the whole angular range, the right panels show an enlargement for small values of $\vartheta$. As expected, the roots of the weight functions are clustered towards lower values of $\vartheta$. Thus, for a fixed maximum number of $n$, these functions resolve those scales better than the linear filter functions. Figure 6 shows the filter functions $W_{n}(\ell)$ which, according to Eq. (5), relates the COSEBIs to the underlying power spectrum $P_{\mathrm{E}}(\ell)$. With increasing $n$, the COSEBIs are sensitive to power at increasingly larger values of $\ell$.

\subsection{E-/B-mode correlation functions}

Crittenden et al. (2002) and Schneider et al. (2002) constructed E-/B-mode correlation functions, which consist of the original correlation function $\xi_{ \pm}(\vartheta)$ plus a correction term which is again an integral over correlation functions. However, these correction terms are unobservable, since the integral extends over an infinite angular range. Thus, these E-/B-mode correlation functions cannot be obtained in practice and are of little use.

With the full E-/B-mode decomposition provided by the COSEBIs, we can define new pure E-/B-mode correlation functions,

$\xi_{ \pm}^{\mathrm{E}}(\vartheta)=\frac{2}{\vartheta \Delta \vartheta} \sum_{n=1}^{\infty} E_{n} T_{ \pm n}(\vartheta)$
$\xi_{ \pm}^{\mathrm{B}}(\vartheta)=\frac{2}{\vartheta \Delta \vartheta} \sum_{n=1}^{\infty} B_{n} T_{ \pm n}(\vartheta)$

obviously, the $\xi_{ \pm}^{\mathrm{E}}$ only depend on the E-mode shear, whereas the $\xi_{+}^{\mathrm{B}}$ contains information only from B-modes. Owing to the constraints (4) which the functions $T_{+n}$ have to obey, one finds that

$\int_{\vartheta_{\min }}^{\vartheta_{\max }} \mathrm{d} \vartheta \vartheta \xi_{+}^{\mathrm{E}}(\vartheta)=0=\int_{\vartheta_{\min }}^{\vartheta_{\max }} \mathrm{d} \vartheta \vartheta^{3} \xi_{+}^{\mathrm{E}}(\vartheta)$

In fact, as shown in SK07, the function $T_{-}$also obeys analogous constraints, namely

$\int_{\vartheta_{\min }}^{\vartheta_{\max }} \frac{\mathrm{d} \vartheta}{\vartheta} T_{-}(\vartheta)=0=\int_{\vartheta_{\min }}^{\vartheta_{\max }} \frac{\mathrm{d} \vartheta}{\vartheta^{3}} T_{-}(\vartheta)$ 

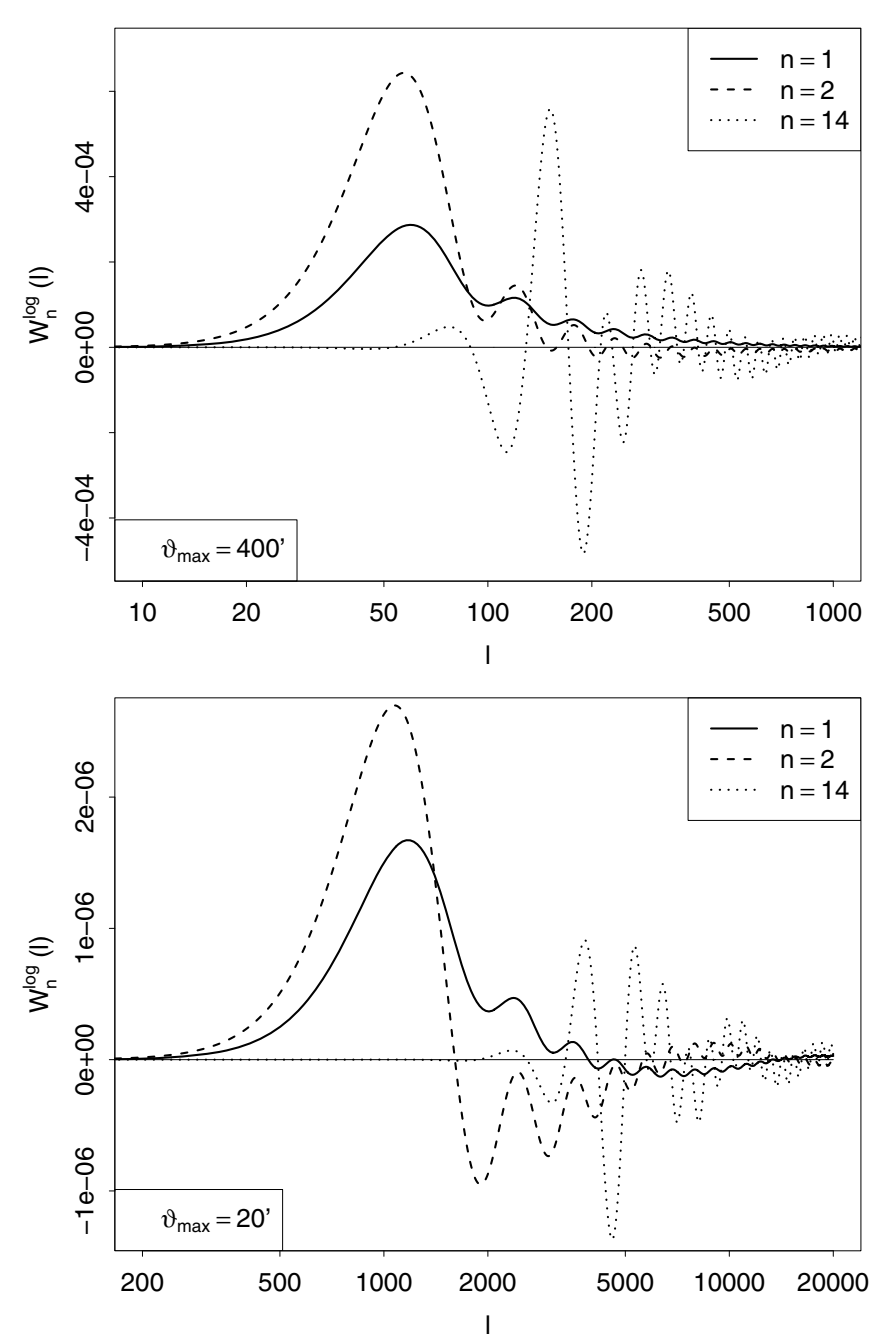

Fig. 6. The $W_{n}$-functions calculated from $T_{n}^{\log }$. The upper panel corresponds to $\vartheta_{\max }=400^{\prime}$, whereas the lower panel is calculated using $\vartheta_{\max }=20^{\prime}$, and $\vartheta_{\min }=1^{\prime}$ in both cases.

so that

$$
\int_{\vartheta_{\min }}^{\vartheta_{\max }} \frac{\mathrm{d} \vartheta}{\vartheta} \xi_{-}^{\mathrm{E}}(\vartheta)=0=\int_{\vartheta_{\min }}^{\vartheta_{\max }} \frac{\mathrm{d} \vartheta}{\vartheta^{3}} \xi_{-}^{\mathrm{E}}(\vartheta) .
$$

In Fig. 7, we have plotted the pure E-mode correlation functions $\xi_{ \pm}^{\mathrm{E}}$, together with the orinial $2 \mathrm{PCFs} \xi_{ \pm}$, for a fiducial $\Lambda \mathrm{CDM}$ cosmological model that will be described in the next section; the overall shape of these functions, however, does not depend on the details of the choice of cosmological parameters. Although not easily visible, $\xi_{ \pm}^{\mathrm{E}}$ both have two roots, as required by the constraints (41) and (42). The function $\xi_{+}^{\mathrm{E}}$ is rather similar in shape to the original $2 \mathrm{PCF} \xi_{+}$, modified in a way as to obey Eq. (41). However, $\xi_{-}^{\mathrm{E}}$ has a very different shape than $\xi_{-}$. In fact, it is easy to see from Eqs. (3) and (4) that $\xi_{-}^{\mathrm{E}}\left(\vartheta_{\text {min }}\right)=\xi_{+}^{\mathrm{E}}\left(\vartheta_{\text {min }}\right), \xi_{-}^{\mathrm{E}}\left(\vartheta_{\max }\right)=\xi_{+}^{\mathrm{E}}\left(\vartheta_{\max }\right)$. In Appendix C, we show how these new pure-mode correlation functions are related to the original 2PCFs. As is obvious from their definition, these pure-mode correlation functions can be obtained from the $2 \mathrm{PCF}$ over a finite interval, hence their estimation does not require extrapolations or "inventing data".

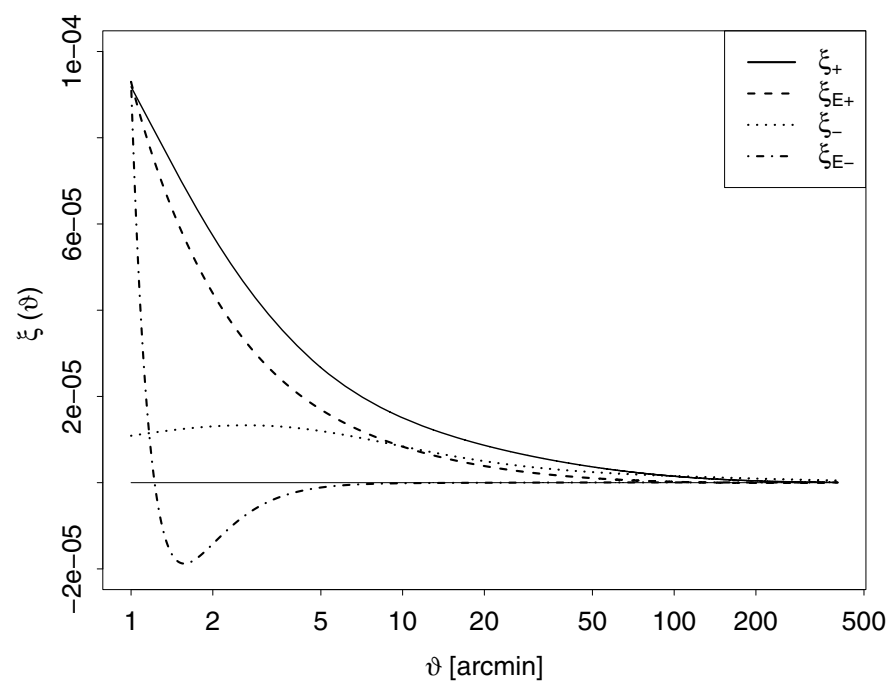

Fig. 7. The $2 \mathrm{PCFs} \xi_{ \pm}(\vartheta)$ and the corresponding pure E-mode correlation functions $\xi_{ \pm}^{\mathrm{E}}(\vartheta)$, for $\vartheta_{\text {min }}=1^{\prime}, \vartheta_{\text {max }}=400^{\prime}$, and the fiducial cosmological model described in Sect. 4.

\section{Likelihood analysis}

We calculate the posterior likelihood in the $\Omega_{\mathrm{m}}-\sigma_{8}$ parameter space for four cases of COSEBIs $\left(\vartheta_{\max }=400^{\prime}, \vartheta_{\max }=20^{\prime}\right.$, each for $T_{n}^{\log }$ and $\left.T_{n}^{\operatorname{lin}}\right)$. Note that, unless stated otherwise, we choose $\vartheta_{\min }=1^{\prime}$ as the minimum separation in the 2PCF. For each of the four cases we are interested in two main questions: first, how does the information content evolve when including more modes $n$ in the likelihood analysis? Second, once it saturates, how large is the difference to the information content of the 2PCFs?

\subsection{Model choice}

In the likelihood analysis we assume a flat universe, and vary the matter density $\Omega_{\mathrm{m}}$ (and simultaneously $\Omega_{\Lambda}=1-\Omega_{\mathrm{m}}$ to preserve flatness) and the normalization $\sigma_{8}$ of the density fluctuations; all other parameters are held fixed, i.e. the dimensionaless Hubble constant $h=0.73$, the density parameter in baryons $\Omega_{\mathrm{b}}=0.044$, and the slope of the primordial fluctuation power spectrum $n_{\mathrm{s}}=1.0$. We choose $\Omega_{\mathrm{m}}=0.27$ and $\sigma_{8}=0.78$ as our fiducial model which enters the likelihood analysis in this section and represents the cosmological model used in Fig. 7. The B-mode power spectrum is set to zero, $P_{\mathrm{B}}(\ell) \equiv 0$, whereas the shear power spectra $P_{\mathrm{E}}$ are obtained from the three-dimensional density power spectra $P_{\delta}$ using Limber's equation (see, e.g., Kaiser 1998). The power spectrum $P_{\delta}$ is calculated with the transfer function from Efstathiou et al. (1992). For the non-linear evolution we use the fitting formula of Smith et al. (2003). In the calculation of $P_{\mathrm{E}}$ we choose a redshift distribution of source galaxies similar to that of Benjamin et al. (2007),

$n(z)=\frac{\beta}{z_{0} \Gamma((1+\alpha) / \beta)}\left(\frac{z}{z_{0}}\right)^{\alpha} \exp \left[-\left(\frac{z}{z_{0}}\right)^{\beta}\right]$,

with $\alpha=0.836, \beta=3.425, z_{0}=1.171$. The corresponding 2PCFs are calculated from Eq. (7), and from these, the COSEBIs are calculated according to Eq. (1) for various modes $n$ using linear and logarithmic filter functions. The covariances used in our likelihood analysis are calculated from the power spectrum $P_{\mathrm{E}}$ as described in Joachimi et al. (2008), assuming our fiducial cosmology. This method does not account for the non-Gaussianity 

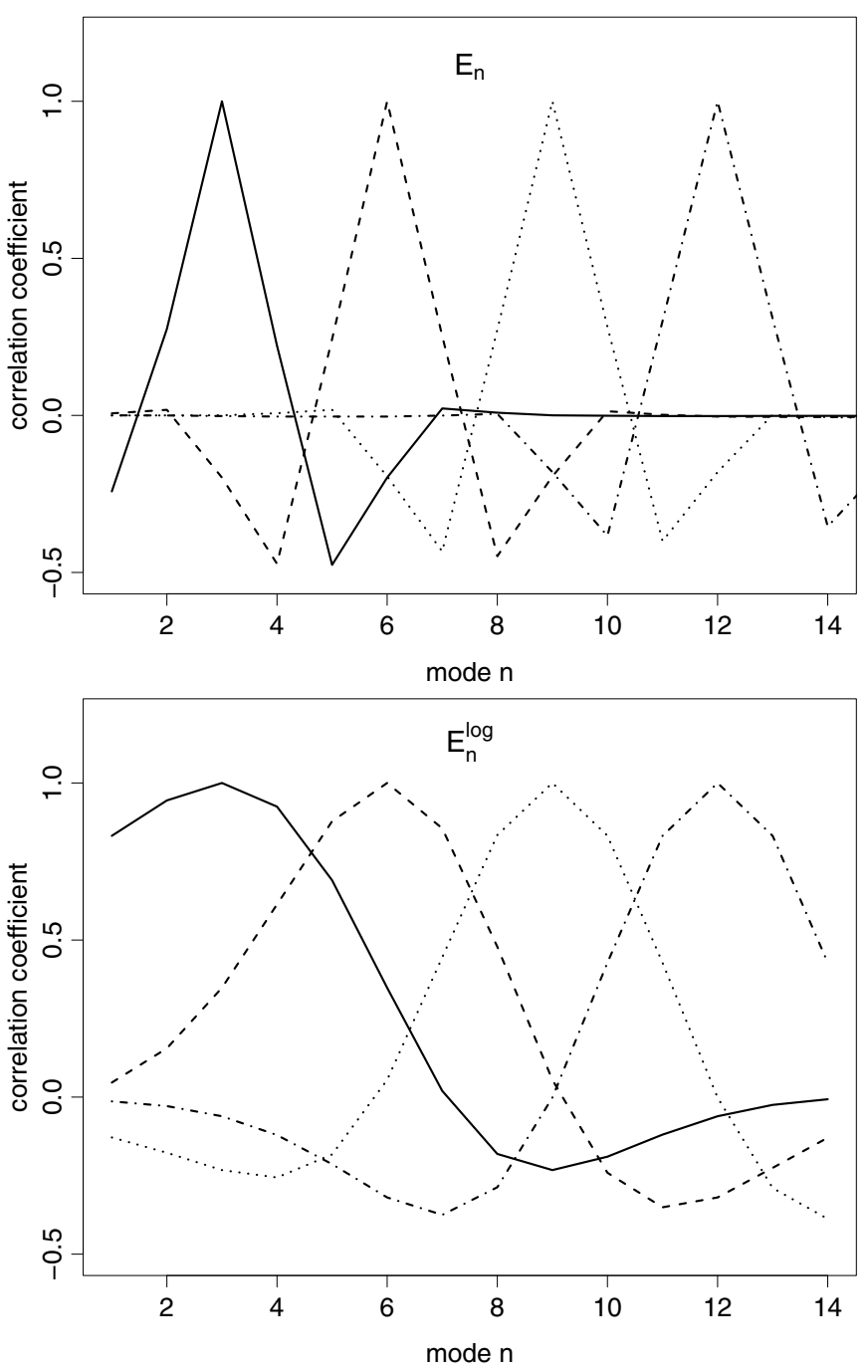

Fig. 8. The correlation coefficients (44) for linear (top) and logarithmic (bottom) weight functions $T_{ \pm n}$, calculated for $\vartheta_{\min }=1^{\prime}, \vartheta_{\max }=400^{\prime}$, and the fiducial cosmological model described in the text.

of the shear field or the cosmology-dependence of the covariance (Eifler et al. 2009), however these issues are not crucial for our purpose as we are only interested in the relative performance of COSEBIs and the 2PCFs. More important is that we can choose an arbitrary binning in the 2PCF covariance. The latter aspect in combination with the speed of the calculation is decisive to resolve the numerical issues in the calculation of the COSEBIs' covariance. The survey parameters read $A=170 \mathrm{deg}^{2}, n_{\text {gal }}=13.3 / \mathrm{arcmin}^{2}$, and $\sigma_{\epsilon}=0.42$, and correspond to those of the upcoming cosmic shear analysis of the full CFHTLS survey area.

The exact method to calculate the posterior likelihood from the data vectors and covariances is described in Eifler et al. (2010). Similar to their analysis we assume flat priors inside the intervals $\Omega_{\mathrm{m}} \in[0.01 ; 1.0]$ and $\sigma_{8} \in[0.4 ; 1.4]$, and zero prior otherwise.

\subsection{The covariance of the COSEBIS}

In Fig. 8 we have plotted the correlation matrix of the COSEBIs, defined as

$r_{m n}=\frac{C_{m n}^{\mathrm{E}}}{\sqrt{C_{m m}^{\mathrm{E}} C_{n n}^{\mathrm{E}}}}$, for several values of $m$, using both linear (upper panel) and logarithmic (lower panel) weight functions. The value of $m$ can be identified as the point where $r_{m n}=1$. For the linear weight functions, we see that the correlation matrix declines quickly for $n \neq m$, reaches a (negative) minimum at $n=m \pm 2$, and essentially is zero for $|m-n| \geq 4$. Thus, the covariance matrix is in essence a band matrix. For the logarithmic COSEBIs, the non-zero correlations between the $E_{n}$ span a larger range in $|m-n|$. One therefore expects that the inversion of the covariance matrix for a given $n_{\max }$ is more difficult for the logarithmic COSEBIs than for the linear ones. However, as we will show below, a smaller number of logarithmic COSEBIs are needed to extract all the cosmological information contained in the shear correlation functions, compared to the linear COSEBIs.

\subsection{Figures of Merit: a short discussion}

In order to illustrate the information content one usually calculates the so-called credible regions, inside of which the true set of parameters is located with a probability of e.g. $68 \%, 95 \%$, $99.9 \%$. Instead of showing likelihood contours for all cases considered, we use two different measures to quantify the size of these credible regions, where each measure characterizes the information contents through a single number.

The first measure, $q$, is calculated from the determinant of the second-order moment of the posterior likelihood $p(\boldsymbol{\pi} \mid \boldsymbol{d})$,

$Q_{i j} \equiv \int \mathrm{d}^{2} \pi p(\boldsymbol{\pi} \mid \boldsymbol{d})\left(\pi_{i}-\pi_{i}^{\mathrm{f}}\right)\left(\pi_{j}-\pi_{j}^{\mathrm{f}}\right)$,

where $\pi_{i}$ are the parameters of the model, and $\pi_{i}^{\mathrm{f}}$ are the parameters of the fiducial model (here, $i=1,2$, corresponding to $\Omega_{\mathrm{m}}$ and $\sigma_{8}$ ). We quantify the size of the credible region by the square root of the determinant of $Q$,

$q=\sqrt{\left|Q_{i j}\right|}=\sqrt{Q_{11} Q_{22}-Q_{12}^{2}}$.

Smaller credible regions in parameter space correspond to smaller values of $q$. In this paper, all $q$ 's are given in units of $10^{-4}$.

Our second figure of merit is obtained from the Fisher information matrix (Tegmark et al. 1997)

$F_{i j}=\frac{1}{2} \operatorname{tr}\left[\mathrm{C}^{-1} \mathrm{C}_{, i} \mathrm{C}^{-1} \mathrm{C}_{, j}+\mathrm{C}^{-1} \mathrm{M}_{i j}\right]$

where subscripts separated by a comma denote partial derivatives with respect to $\pi_{i}$, and $\mathbf{M}_{i j}=\mathbf{E}_{, i} \mathbf{E}^{\mathbf{t}}{ }_{j}+\mathbf{E}_{, j} \mathbf{E}^{\mathbf{t}}{ }_{, i}$, where $\mathbf{E}$ is the $n_{\max }$-dimensional vector of the first $n_{\max } E_{n}$ 's. The $n_{\max } \times n_{\max }{ }^{-}$ dimensional covariance matrix $C$ has the elements $C_{m n}^{\mathrm{E}}$, as given in Eq. (8). We consider a constant covariance in parameter space, so that the first term of Eq. (47) vanishes. Since the Fisher matrix is the Hessian of the (negative of the) log-likelihood function at its maximum, its elements describe the size and shape of ellipses of constant likelihood near the maximum. If the likelihood was strictly Gaussian, the Fisher matrix would completely describe its functional form. We define our second figure of merit $f$ as

$f=\frac{1}{\sqrt{\operatorname{det}(\mathrm{F})}}$.

For a better comparison with $q$ we chose to modify the more commonly used figure of merit definition (see e.g., Albrecht et al. 2006) - we consider the area of the error ellipse itself, not its inverse. Similar to $q, f$ is given in units of $10^{-4}$. With the 


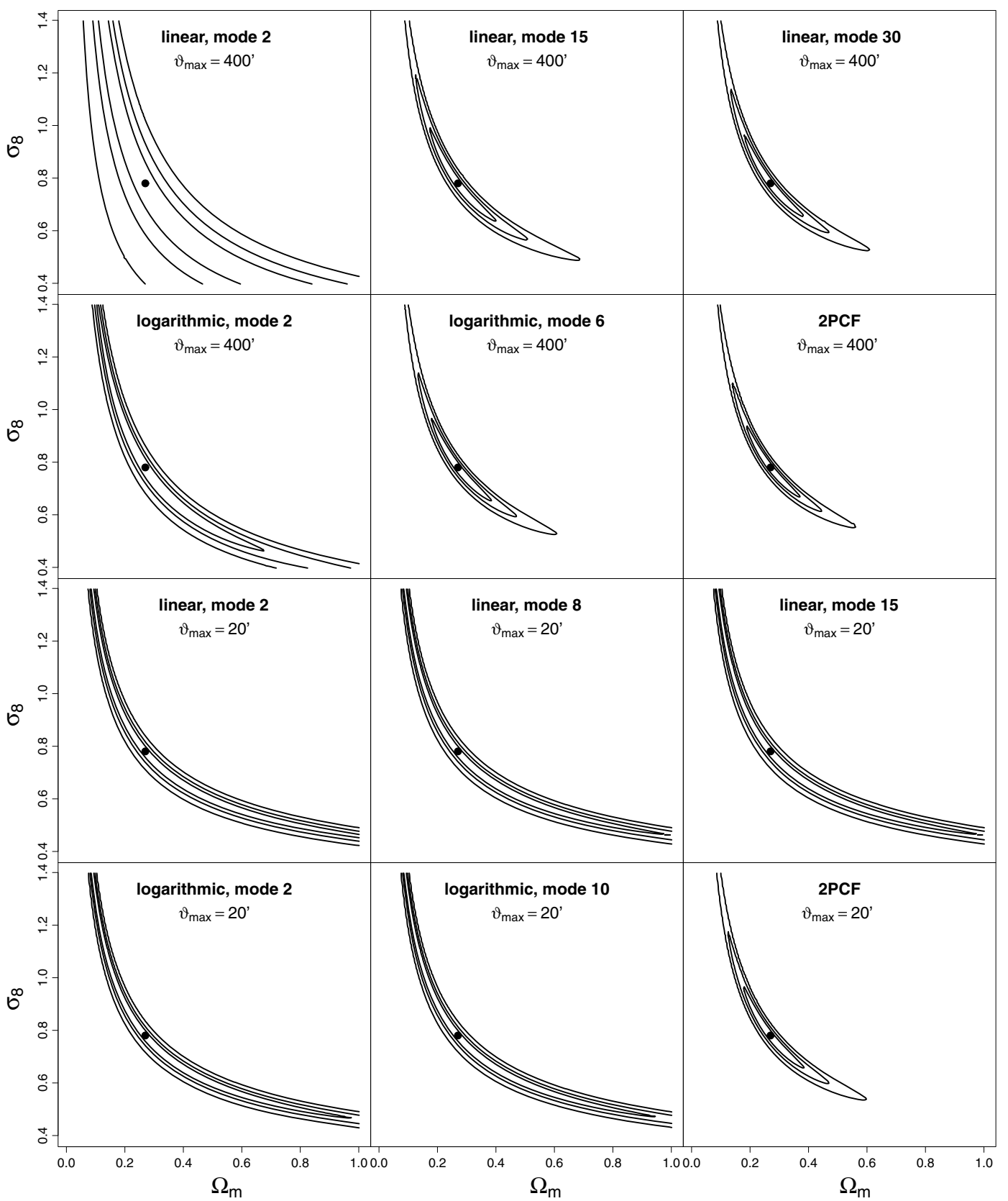

Fig. 9. Likelihood contours for a fiducial cosmic shear survey, with parameters described in Sect. 4.1. The upper (lower) six panels correspond to $\vartheta_{\max }=$ $400^{\prime}\left(20^{\prime}\right)$. Shown in the first and third rows are the likelihood as obtained from the COSEBIs with linear filter functions and various $n_{\max }$, in the second and fourth rows the likelihood as obtained from the logarithmic filter functions, and in comparison, we show the likelihood obtained from the shear two-point correlation functions. definition (48), $q$ and $f$ give the same result if (1) the likelihood in the parameter space considered is Gaussian and (2) if the likelihood outside the region where we set a flat prior is negligible. We note that $f$ and $q$ can be significantly different if these two assumptions are not satisfied. Then, the Gaussian defined by the Fisher matrix is only a useful approximation close to the fiducial model, and the resulting values of $f$ can be rather bad approximations for $q$. In contrast, $q$ is sensitive to parameter regions far from the fiducial model and we therefore consider $q$ as the more useful measure for the information contents. In order to give an impression of the meaning of different $q$ and $f$ we show a sample of likelihood contours in Fig. 9 - it is obvious that the likelihood function in our case is far from Gaussian.

\subsection{Results of the likelihood analysis}

Figure 10 shows the values of $q$ for the case of $\vartheta_{\max }=400^{\prime}$ (left panel) and for $\vartheta_{\max }=20^{\prime}$ (right panel). The triangles correspond to the COSEBIs from $T_{n}^{\log }$, whereas the circles correspond to the COSEBIs calculated using the linear $T_{n}$. For comparison we show the information content of the 2PCF (dashed line), which serves as an upper limit on the information content of any second-order E/B-decomposing measure - since the 2PCFs contain all information from second-order shear measurements and the COSEBIs are derived from them (Eifler et al. 2008).

For $\vartheta_{\max }=400^{\prime}$ the minimum value of $q$ obtainable from the COSEBIs - and thus the available information on the two cosmological parameters considered - is extremely close to that obtained from the 2PCFs. The logarithmic $E_{n}$ reach this threshold already for $n_{\max }=5$, whereas the linear $E_{n}$ saturate around $n_{\max }=25$, indicating that the logarithmic modes capture the bulk of cosmological information in significantly fewer data points compared to the linear case. This property can be particularly important in higher-dimensional parameter spaces, where data compression and computing time become important.

The COSEBIs for $\vartheta_{\max }=20^{\prime}$ saturate much earlier; the information content of $E_{n}$ is hardly increased when going beyond 
P. Schneider et al.: COSEBIs: Extracting the full E-/B-mode information from cosmic shear correlation functions
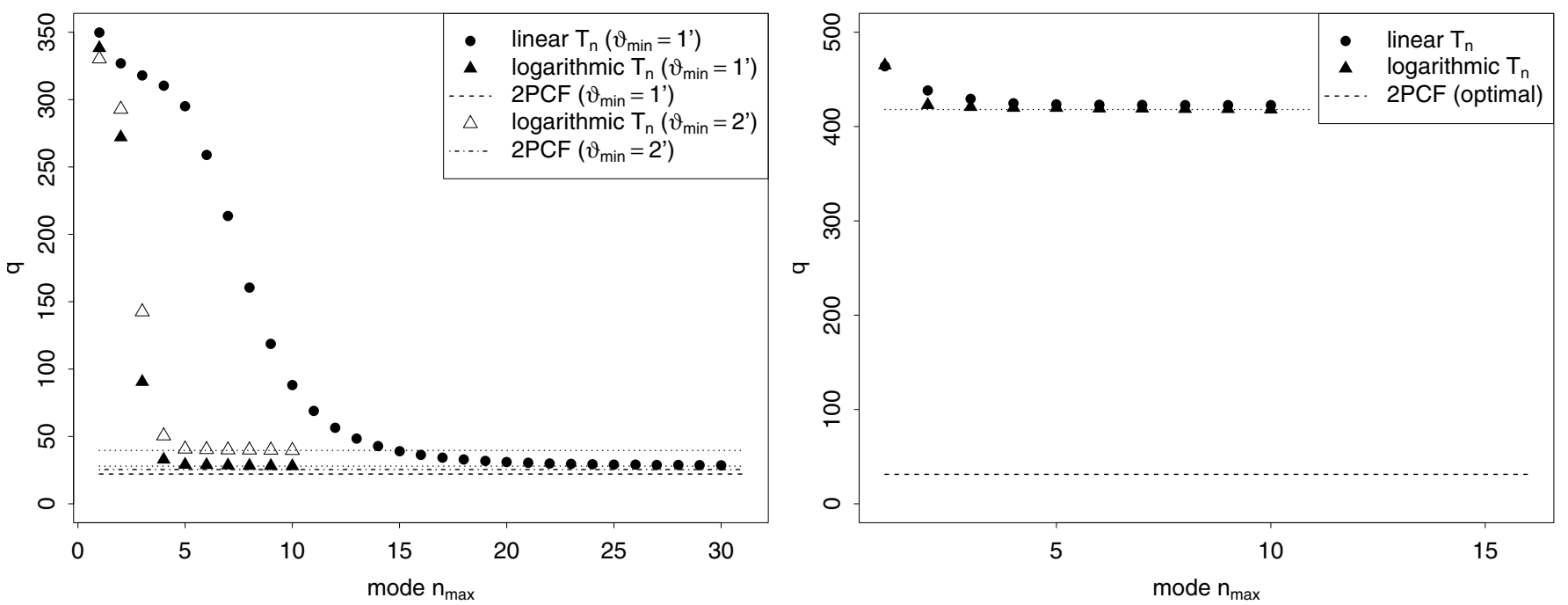

Fig. 10. The values of $q$ - see Eq. (46) - calculated from the COSEBIs for the case of linear (circles) or logarithmic (triangles) $T_{n}$-functions, as a function of the maximum mode $n_{\max }$ which was included in the likelihood analysis. The results in the left (right) panel correspond to $\vartheta_{\max }=400^{\prime}$ $\left(\vartheta_{\max }=20^{\prime}\right)$, and the filled symbols are calculated for $\vartheta_{\min }=1^{\prime}$; in the left panel, we also plot corresponding results for $\vartheta_{\min }=2^{\prime}$, indicated by the open triangles. The dashed (dash-dotted) line represents the optimal $q$ for $\vartheta_{\min }=1^{\prime}\left(\vartheta_{\min }=2^{\prime}\right)$, obtained when using the $2 \mathrm{PCFs}$ directly. The dotted lines shows the asymptotic value of $q$ achieved for large $n_{\max }$.

$n=4$ ( $n=3$ for the logarithmic weight functions). More important, however, is the large difference between the saturation limit of the COSEBIs and the corresponding information content of the 2PCFs (which is also seen in the likelihood contours of Fig. 9). Obviously, the choice of $\vartheta_{\max }$ has a significant impact on the information content, and on the relative information contained in the COSEBIs and the 2PCFs.

This latter difference is not due to a deficiency of the COSEBIs - since they form a complete set of E-/B-mode measures, they contain all the information that can uniquely be split into the two modes. If, however, one assumes that the shear field has no B-mode contribution, and thus using of the full 2PCFs obviously yields tighter parameter constraints. But, this assumption will hardly be justifiable in any of the forthcoming surveys. The fact that the measured B-modes are compatible with zero within the error bars in a data set is not a justification - since any realistic survey may contain B-modes which cannot be identified as such, for example a uniform shear field which can either be E- or B-mode. Therefore, the loss of information due to a clean mode separation is inevitable, but a small price to pay relative to a potential bias of results due to undetected B-modes. Fortunately, for surveys which allow shear correlation measurements on large angular scales, this information loss is seen to be almost negligible.

We analyse this more closely in Fig. 11, where we show $q$ as a function of $\vartheta_{\max }$; here we use logarithmic weight functions with 10 modes, i.e., where the asymptotic limit is well achieved. The amount of information increases significantly when going from $20^{\prime}$ to $100^{\prime}$ and becomes almost constant when going to larger $\vartheta_{\text {max }}$. This behavior, of course, depends on the parameter space considered; for $\Omega_{\mathrm{m}}-\sigma_{8}$ it can be understood from the functional behavior of the power spectrum. For small $\ell$, it is almost fully degenerate in these two parameters, hence going to larger angular scales does not yield significantly more information - this will be different for other parameter combinations. One also sees that the difference in information content between the COSEBIs and the 2PCFs decreases for larger $\vartheta_{\max }$ - the larger $\vartheta_{\max }$, the smaller is the contribution of modes to the 2PCFs which can not be uniquely decomposed into E/B-modes.

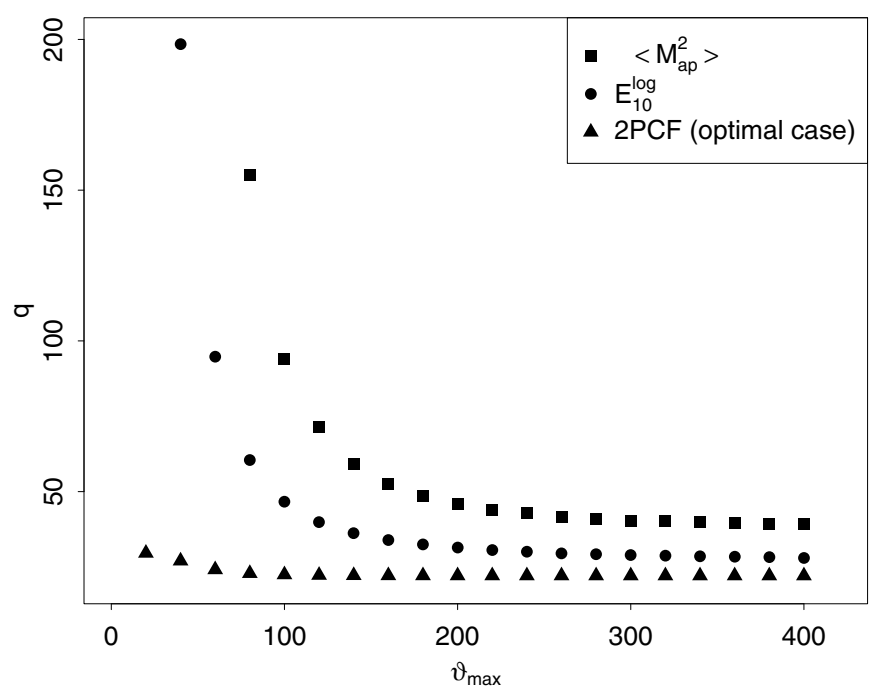

Fig. 11. The $q$ of the COSEBIs as a function $\vartheta_{\max }$, for $\vartheta_{\min }=1^{\prime}$. The COSEBIs are calculated from $T_{n}^{\log }$, where $n$ ranges from 1-10.

Furthermore, we have plotted the corresponding values of $q$ for the aperture dispersion $\left\langle M_{\mathrm{ap}}^{2}(\theta)\right\rangle$, where $\theta=\vartheta_{\max } / 2$ is the aperture radius which is calculated from the shear $2 \mathrm{PCF}$ for $\vartheta \leq \vartheta_{\max }$. Values for $\left\langle M_{\mathrm{ap}}^{2}(\theta)\right\rangle$ are calculated and plotted only for $\theta \geq 40^{\prime}$, to limit the bias caused by the lack of measured correlation functions for $\vartheta<\vartheta_{\text {min }}$ (see Kilbinger et al. 2006) to $<5 \%$. We see that its information content is smaller than that of the COSEBIs, as it must be the case, owing to the completeness of the latter.

Figure 12 shows a similar analysis based on $f$. The results confirm our foregoing findings. Similar to the case of $q$, the Fisher matrix analysis shows that the logarithmic $E_{n}$ reach the saturation limit much earlier than the linear $E_{n}$ and again, the saturation limit for $\vartheta_{\max }=400^{\prime}$ is closer to the optimal information content than for $\vartheta_{\text {max }}=20^{\prime}$. 

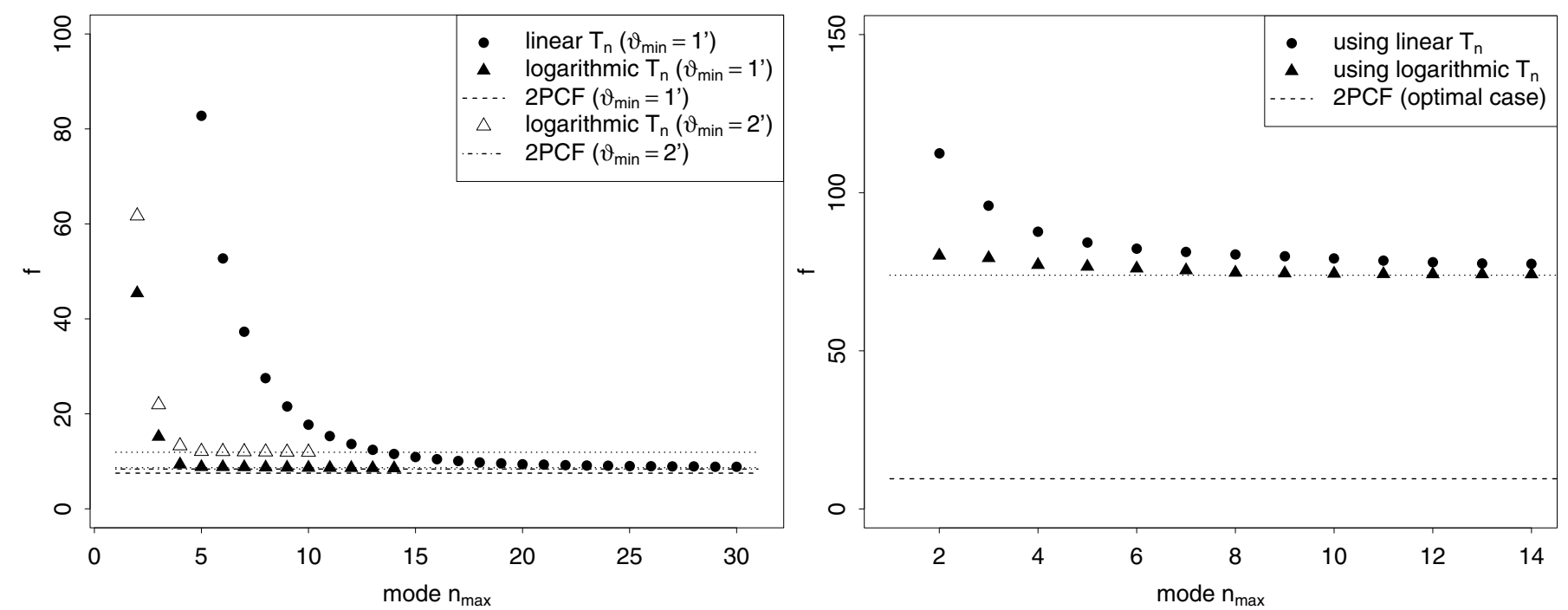

Fig. 12. The value of $f$ - see Eq. (48) - for the case of linear (circles) or logarithmic (triangles) $T_{n}$-functions as a function of the maximum mode $n_{\max }$ which was included in the likelihood analysis. The results in the left (right) panel correspond to $\vartheta_{\max }=400^{\prime}\left(\vartheta_{\max }=20^{\prime}\right)$, and the filled symbols are calculated for $\vartheta_{\min }=1^{\prime}$; in the left panel, we also plot corresponding results for $\vartheta_{\min }=2^{\prime}$, indicated by the open triangles. The dashed (dash-dotted) line represents the optimal $f$ for $\vartheta_{\min }=1^{\prime}\left(\vartheta_{\min }=2^{\prime}\right)$, obtained when using the 2PCFs directly. The dotted lines shows the asymptotic value of $f$ achieved for large $n_{\max }$.

Table 1. Values of $q$ and $f$ as obtained by considering the full 2PCFs, and by using the COSEBIs $E^{\text {lin }}$, and $E^{\log }$

\begin{tabular}{lllll}
\hline \hline Measure & $\vartheta_{\min }$ & $\vartheta_{\max }$ & $q$ & $f$ \\
\hline $2 \mathrm{PCF}$ & $1^{\prime}$ & $400^{\prime}$ & 22.10 & 7.51 \\
$E_{30}^{\operatorname{lin}}$ & $1^{\prime}$ & $400^{\prime}$ & 28.68 & 8.85 \\
$E_{10}^{\log }$ & $1^{\prime}$ & $400^{\prime}$ & 27.04 & 8.66 \\
& & & & \\
$2 \mathrm{PCF}$ & $1^{\prime}$ & $20^{\prime}$ & 31.28 & 9.54 \\
$E_{15}^{\operatorname{lin}}$ & $1^{\prime}$ & $20^{\prime}$ & 422.79 & 74.21 \\
$E_{10}^{\log }$ & $1^{\prime}$ & $20^{\prime}$ & 418.09 & 77.35 \\
& & & & \\
$2 \mathrm{PCF}$ & $2^{\prime}$ & $400^{\prime}$ & 25.46 & 8.37 \\
$E_{10}^{\log }$ & $2^{\prime}$ & $400^{\prime}$ & 39.65 & 11.91 \\
\hline
\end{tabular}

Note that in Figs. 10 and 12 we choose a similar scale for the vertical axis in the right and the left panels to enable for an easier comparison between the different cases of $\vartheta_{\text {max }}$. We point out the good agreement between the saturation limits of $E_{n}$ and $E_{n}^{\log }$ in all cases, which shows that our results are numerically robust. In Table 1, we have listed the values of $q$ and $f$ as shown in Figs. 10 and 12 for the maximum number $n_{\max }$ of modes. The small difference between these values as obtained from the linear and logarithmic weight functions for the COSEBIs is due to the fact that for these values of $n_{\max }$, the linear ones have not yet reached their full asymptotic value.

The underlying reason why the formal loss of information of the COSEBIs, relative to the full 2PCFs, is larger for smaller $\vartheta_{\max }$ is due to the filter functions that relates the 2PCFs to the power spectrum. This filter function is $J_{0}(x)$ for the case of $\xi_{+}(\vartheta)$, i.e. a function that tends towards +1 for small arguments. This implies that the correlation function $\xi_{+}(\vartheta)$ is sensitive to long-range modes, i.e., modes of small $\ell$. In particular, this means that $\xi_{+}$is also sensitive to the power spectrum for modes satisfying $\ell \leq 2 \pi / \vartheta_{\max }$, corresponding to scales which are in fact not probed by the $2 \mathrm{PCF}$ directly - and for which no E-/B-mode separation is possible from the data. The relative cosmological information content of the power spectrum in the ranges $\ell \leq 2 \pi / \vartheta_{\max }$ and $2 \pi / \vartheta_{\max } \lesssim \ell \lesssim 2 \pi / \vartheta_{\min }$ decreases with increasing $\vartheta_{\max }$, which explains the difference in "relative information loss" in Figs. 10 through 12.

Up to now we have always chosen $\vartheta_{\min }=1^{\prime}$. However, one may ask whether cosmic shear measurements down to this angular scale can be compared to sufficient accuracy with cosmological predictions, since at the corresponding length scales, baryonic physics can have a significant influence on the projected power spectrum. Of course, modeling the behavior of baryons in a cosmological simulation is much more difficult, and burdened with higher uncertainty, than dark matter-only simulations. Jing et al. (2006) compared pure dark matter simulations with hydrodynamic simulations to conclude that for $\ell \sim 10^{4}$, corresponding to $\vartheta \sim 1^{\prime}$, the predicted power spectra differ by about $10 \%$ - much more than the predicted statistical uncertainty of future cosmic shear surveys.

Fortunately, the largest effect of baryons on the total mass distribution seems to be a change of the halo concentration parameter as a function of halo mass (Rudd et al. 2008), in that baryons render halos more concentrated. If this is the case, then this effect can be calibrated from the weak lensing data themselves. Zentner et al. (2008) studied such a self-calibration method for future surveys and concluded that the concentrationmass relation can be determined from the weak lensing data. In the framework of the halo model for the large-scale structure, the power spectrum can then be calculated using this modified concentration-mass relation, and fairly accurate model predictions can be made.

Dropping the small angular scales from future surveys implies considerably weaker cosmological constraints. In the left panels of Figs. 10 and 12, we have plotted the values of $q$ and $f$, respectively, for surveys with $\vartheta_{\text {min }}=2^{\prime}$. Independent of whether the "optimal" constraints from the 2PCFs or the COSEBIs are employed, the resulting constraints are weaker than for $\vartheta_{\text {min }}=1^{\prime}$. Therefore, it is of considerable interest to 
improve the accuracy of predictions for the matter power spectrum to small scales, to make full use of the information contained in cosmic shear surveys on small angular scales.

\section{Summary and discussion}

We have defined pure E- and B-mode cosmic shear measures from correlation functions over a finite interval $\vartheta_{\min } \leq \vartheta \leq \vartheta_{\max }$. These are complete orthonormal sets of such measures, implying that they contain all cosmic shear information in the two-point correlation functions which can be uniquely split into E- and B-modes. For these COSEBIs, we have calculated their relation to the underlying power spectrum and their covariance matrix. Two different sets of COSEBIs have been explicitly constructed, those with weight functions which are polynomials in the angular scale, and those with polynomial weight functions in the logarithm of the angular scale. For the former case, analytic expressions were obtained for all orders, whereas in the logarithmic case, a linear system of equations needs to be solved numerically.

\subsection{Advantages of the COSEBIS}

Comparing the COSEBIs with earlier cosmic shear measures, we point out a number of advantages. First, using the correlation functions themselves does not provide an E-/B-mode separation. The construction of E-/B-mode correlation functions as described in (Crittenden et al. 2002) requires knowledge of the correlation functions over an infinite angular range, and is therefore not applicable in practice (extrapolating to infinite separation using fiducial cosmological models corresponds to "inventing data", and implicitly assumes that there are no long-range B-modes). In fact, the generalization of pure E-/B-mode correlation functions based on data over a finite angular range has been derived here (see Sect. 2 and Appendix C); however, we expect these to be of limited use in practice.

Whereas the aperture mass dispersion (Schneider et al. 1998) provides a clean separation into E- and B-modes (Crittenden et al. 2002; Schneider et al. 2002), it requires the knowledge of the correlation function to arbitrarily small angular separation. There are at least two aspects which render this impractical: first, galaxy images need a minimum separation for their shapes to be measurable. Second, on very small scales baryonic effects will affect the power spectrum and render model predictions very uncertain. The inevitable bias of the aperture mass dispersion (Kilbinger et al. 2006) motivated the ring statistics (Schneider $\&$ Kilbinger 2007). The latter removes the bias, depends only on the correlation function over a finite interval, and has potentially higher sensitivity to cosmological parameters (Eifler et al. 2010, FK10). However, the weight function of the ring statistics is largely arbitrary.

The COSEBIs contain all available mode-separable information from the correlation functions on a finite interval, and are therefore guaranteed to provide highest sensitivity to cosmological parameters. Furthermore, they form a discrete set of measures, whereas the other cosmic shear statistics include a somewhat arbitrary grid of variables, like the outer scale of the ring statistics: if the grid is too coarse, information gets lost, whereas a finer grid renders the measures largely redundant, implying large and significantly non-diagonal covariances. In contrast, the discreteness of COSEBIs leaves no freedom, and for the linear weight functions, the covariances have a narrow band structure. The information clearly saturates after a number of modes, and this number is surprisingly small for the logarithmic weight function. Therefore, determining covariance matrices from numerical simulations (as was done for the COSMOS analysis of Schrabback et al. 2009) appears considerably simpler than for other cosmic shear measurements, which is particularly true for an unbiased estimate of their inverse (see Hartlap et al. 2007, for a discussion of this point). Based on these properties of the COSEBIs, we would like to advertise them as the method of choice for future cosmic shear analyses.

\subsection{Generalizations}

In case photometric redshift information of the lensed galaxies is available and several source populations can be defined based on their redshift estimates, the COSEBIs can be generalized to a tomographic version. Furthermore, under the same assumption, intrinsic alignment effects between the tidal gravitational field and the intrinsic galaxy orientation (e.g., Catelan et al. 2001; Crittenden et al. 2001; Jing 2002; Hirata \& Seljak 2004) can be filtered out by properly choosing redshift-dependent weight functions, such as to avoid physically close pairs of galaxies (King \& Schneider 2002, 2003; Heymans \& Heavens 2003) or make use of the specific redshift dependence of the shearintrinsic alignments (Bridle \& King 2007; Joachimi \& Schneider 2008, 2009), possibly in combination with other data (Joachimi \& Bridle 2009). We expect that these generalizations of the COSEBIs provide no real difficulties.

It would be desirable to obtain a similar measure for thirdorder cosmic shear statistics, i.e., one that provides clear E-/Bmode separations from three-point correlation functions measured over a finite interval. Up to now, the aperture statistics is the only known such measure (Jarvis et al. 2004; Schneider et al. 2005); however, similar to the case of the aperture dispersion, third-order aperture statistics requires the correlation functions to be measured down to arbitrarily small separations. A generalization of the COSEBIs to third order seems challening not only because of the higher number of independent variables (the three-point correlation functions depend on three variables) and the larger number of modes (one pure E-mode, one mixed E/B-mode, and two further modes which are not invariant under parity transformation), but also because of the more complicated relation between correlation functions and the bispectra (Schneider et al. 2005). Thus, even the analogue of the starting point of the current paper - Eqs. (1) and (3) - is not yet known for the third-order case.

Acknowledgements. We thank Liping Fu and Martin Kilbinger for interesting discussions on E-/B-mode separations which triggered this study, and an anonymous referee for constructive suggestions. We thank Marika Asgari for checking some of the numerical results presented here. This work was supported by the Deutsche Forschungsgemeinschaft within the Transregional Research Center TR33 "The Dark Universe" and the Priority Programme 1177 "Galaxy Evolution" under the project SCHN 342/9.

\section{Appendix A: Calculation of the COSEBIs: numerical problems and solutions}

Several numerical issues arose during the implementation of the calculations of the COSEBIs, especially in the context of their covariance. As these issues are crucial for obtaining the correct values of $q$ and $f$, we outline them in greater detail. We employ the QAG adaptive integration routine from the GNU Scientific Library ${ }^{1}$ and obtain the $E_{n}$ using two different methods. First, we calculate them from the set of 2PCFs according to Eq. (1) and second, we check for consistency by calculating $E_{n}$ directly from

${ }^{1}$ http://www.gnu.org/software/gsl/ 
the $P_{\mathrm{E}}$ according to Eq. (5). The first method cleanly separates E- and B-modes, giving a B-mode residual due to numerical uncertainties which is 8 to 5 orders of magnitudes lower than the E-mode, depending on the scales considered and whether one uses $T_{n}$ or $T_{n}^{\log }$. Both methods yield results in perfect agreement, hence we are confident that there are no numerical problems in either of them.

When using a binned version of the $2 \mathrm{PCF}$ instead, we find a non-negligible deviation when using too few angular bins. The number of bins, above which the E/B-decomposition becomes stable, depends on the mode $E_{n}$, the maximum scale $\vartheta_{\text {max }}$ of the 2PCF, and whether one uses $T_{n}$ or $T_{n}^{\log }$. This should be checked carefully before applying the method to an actual data set. As an example, we found that for linear $T_{n}$ and $\vartheta_{\max }=400^{\prime}$, one needs $\sim 10^{5}$ bins to calculate $E_{30}$ properly and to have an accurate mode separation.

The calculation of the covariance $C_{m n}^{\mathrm{E}}$ is numerically more challenging than that of the data vectors. Again, we use two approaches and calculate $\mathrm{C}^{\mathrm{E}}$ from $P_{\mathrm{E}}$ using Eq. (8), and from the 2PCF covariance using Eq. (9). Both methods have their difficulties and need to be checked carefully for consistency before using the covariance in the likelihood analysis.

The power spectrum approach using Eq. (5) involves the calculation of a one-dimensional integral over $P_{\mathrm{E}}$ multiplied by two filter functions $W_{ \pm}$. As can be seen from Figs. 2 and 6, these filter functions are strongly oscillating, which becomes worse for large $\ell$ and higher modes $n$. We use a stepwise integration to calculate the integral and truncate the integral once the ratio of "new contribution in step $i$ /integral calculated until $(i-1)$ " drops below a certain threshold. We vary the width of the steps as well as the truncation threshold; however, we find that the integration becomes inaccurate when going to higher modes $n$.

For calculation of $C_{m n}^{\mathrm{E}}$ from the covariance of the 2PCFs we find that it is too time-consuming to calculate the 2PCF covariance for every sampling point of the integration routine separately. Instead, we calculate the $2 \mathrm{PCF}$ covariance for a specific binning and interpolate the values during the integration. We use a linear binning in the $2 \mathrm{PCF}$ covariance for the linear weight function and a logarithmic binning for the case of $T_{n}^{\log }$. In addition, we check how strongly the number of bins influences the accuracy of the integral, finding that we can calculate $\mathrm{C}^{\mathrm{E}}$ properly if we choose at least $1000 \times 1000$ bins in the 2 PCF covariance. The final $\mathrm{C}^{\mathrm{E}}$ must be symmetric, positive definite, and not ill-conditioned, as already small deviations from these requirements can bias the information content measures $q$ and $f$.

\section{Appendix B: S/N maximization}

From a complete set of functions $T_{+n}$ obeying the constraints (4) for given $\vartheta_{\text {min }}$ and $\vartheta_{\max }$, we can find a weight function $T_{+}(\vartheta)$ which maximizes the signal-to-noise of the E-mode. This problem was also considered by FK10. In this case, we can write

$T_{+}(\vartheta)=\sum_{n=1}^{N} a_{n} T_{+n}(\vartheta)$

which satisfies the integral constraints (4) for any choice of the $a_{n}$. Then the E-mode signal is, in the absence of B-modes,

$E=\int_{\vartheta_{\min }}^{\vartheta_{\max }} \mathrm{d} \vartheta \vartheta T_{+}(\vartheta) \xi_{+}(\vartheta)=\sum_{n=1}^{N} a_{n} E_{n}$.
The noise $N$ of $E$ is obtained through the covariance of the $E_{n}$,

$N^{2}=\left\langle E^{2}\right\rangle-\langle E\rangle^{2}=\sum_{m, n=1}^{N} a_{m} a_{n} C_{m n}^{\mathrm{E}}$,

yielding as signal-to-noise ratio

$\frac{S}{N}=\frac{\sum_{n} a_{n} E_{n}}{\sqrt{\sum_{m, n} a_{m} a_{n} C_{m n}^{\mathrm{E}}}}$.

To obtain a maximum of $S / N$ with respect to the coefficients $a_{n}$, we differentiate the foregoing expression with respect to a coefficient $a_{k}$,

$$
\begin{aligned}
\frac{\partial}{\partial a_{k}} \frac{S}{N}= & \frac{E_{k}}{\sqrt{\sum_{m, n} a_{m} a_{n} C_{m n}^{\mathrm{E}}}} \\
& -\frac{\sum_{n} a_{n} E_{n}}{2 N^{3}} \sum_{m, n}\left(\delta_{m k} a_{n} C_{m n}^{\mathrm{E}}+\delta_{n k} a_{m} C_{m n}^{\mathrm{E}}\right) \\
= & N^{-3}\left[N^{2} E_{k}-\left(\sum_{n} a_{n} E_{n}\right)\left(\sum_{n} C_{k n}^{\mathrm{E}} a_{n}\right)\right] .
\end{aligned}
$$

Setting this derivative to zero results in

$$
E_{k}=\frac{\sum_{n} a_{n} E_{n}}{\sum_{m, n} a_{m} a_{n} C_{m n}^{\mathrm{E}}} \sum_{n} C_{k n}^{\mathrm{E}} a_{n} .
$$

From this equation we see that the overall amplitude of the $a_{n}$ cannot be determined, i.e., if the $a_{n}$ are a solution, then $\lambda a_{n}$ solve the equation as well. Noting that the first term on the r.h.s. of Eq. (B.6) does not depend on $k$, a solution is obtained as

$a_{k}=\sum_{n}\left(C^{\mathrm{E}}\right)_{k n}^{-1} E_{n}$

as can be also verified by inserting this into Eq. (B.6). Thus, if the function $T_{+}$is expanded into a set of functions which all satisfy the constraints (4), the signal-to-noise maximization can be done analytically. If different sets of functions are used for constructing the $T_{+}$maximizing the $S / N$, the resulting function should be the same in the limit $N \rightarrow \infty$; however, different sets of functions may require different $N$ before the asymptotic limit is reached.

\section{Appendix C: Pure E-/B-mode correlation functions}

We will now explore how the pure-mode correlation functions introduced in Eq. (40) are related to the original $\xi_{ \pm}$. For this, we use Eq. (1) in the definition (40) to obtain

$$
\begin{aligned}
\xi_{ \pm}^{\mathrm{E}, \mathrm{B}}(\vartheta)= & \int_{\vartheta_{\min }}^{\vartheta_{\max }} \frac{\mathrm{d} \varphi \varphi}{\vartheta \Delta \vartheta}\left[\xi_{+}(\varphi) \sum_{n=1}^{\infty} T_{ \pm n}(\vartheta) T_{+n}(\varphi)\right. \\
& \left.+\mu \xi_{-}(\varphi) \sum_{n=1}^{\infty} T_{ \pm n}(\vartheta) T_{-n}(\varphi)\right] \\
= & \int_{\vartheta_{\min }}^{\vartheta_{\max }} \frac{\mathrm{d} \varphi \varphi}{\vartheta \Delta \vartheta}\left[\xi_{+}(\varphi) S_{ \pm+}(\vartheta, \varphi)+\mu \xi_{-}(\varphi) S_{ \pm-}(\vartheta, \varphi)\right]
\end{aligned}
$$

where $\mu=+1$ for E-modes, $\mu=-1$ for the B-modes, and where we defined the functions $S_{ \pm \pm}(\vartheta, \varphi)$ in the last step. These functions are calculated next, by noting that the normalized Legendre polynomials $p_{n}(x)$ as defined in Eq. (19) are orthonormal,

$\int_{-1}^{1} \mathrm{~d} x p_{n}(x) p_{m}(x)=\delta_{m n}$ 
P. Schneider et al.: COSEBIs: Extracting the full E-/B-mode information from cosmic shear correlation functions

form a complete set of functions on the interval $[-1,1]$, and therefore obey

$\sum_{n=0}^{\infty} p_{n}(x) p_{n}(y)=\delta_{\mathrm{D}}(x-y)$.

Noting that we have chosen in Sect. $3.1 t_{n}(x)=p_{n+1}(x)$ for $n \geq 3$, we find that

$$
\begin{aligned}
s_{++}(x, y) & =\sum_{n=1}^{\infty} t_{+n}(x) t_{+n}(y) \\
& =\sum_{n=4}^{\infty} p_{n}(x) p_{n}(y)+\sum_{n=1}^{2} t_{+n}(x) t_{+n}(y) \\
& =\delta_{\mathrm{D}}(x-y)-\sum_{n=0}^{3} p_{n}(x) p_{n}(y)+\sum_{n=1}^{2} t_{+n}(x) t_{+n}(y) \\
& =: \delta_{\mathrm{D}}(x-y)-F_{++}(x, y),
\end{aligned}
$$

where in the final step we have defined the function $F_{++}(x, y)$, which is obviously symmetric in its arguments. The explicit expression for it reads

$$
\begin{aligned}
& F_{+}(x, y)=\frac{5(1+B x)(1+B y)}{8\left(175+35 B^{2}+45 B^{4}+B^{6}\right)} \\
& \quad \times(140(1+3 x y)+70 B(3 x y-5)(x+y) \\
& \quad+7 B^{2}\left[39+20 x y-25\left(x^{2}+y^{2}\right)+15 x^{2} y^{2}\right] \\
& \left.\quad+14 B^{3}(5 x y-3)(x+y)+B^{4}\left[15-21\left(x^{2}+y^{2}\right)+35 x^{2} y^{2}\right]\right) .
\end{aligned}
$$

We can now calculate the other sums in Eqs. (C.1), making use of Eq. (20) written in the form

$t_{-n}(x)=t_{+n}(x)+\int_{-1}^{x} \mathrm{~d} z t_{+n}(z) \mathcal{G}(z, x)$,

with

$\mathcal{G}(z, x)=\frac{4 B}{(1+B x)^{2}}\left[1+B z-\frac{3(1+B z)^{3}}{(1+B x)^{2}}\right]$.

This then yields

$$
\begin{aligned}
s_{+-}(x, y) & =\sum_{n=1}^{\infty} t_{+n}(x) t_{-n}(y) \\
& =s_{++}(x, y)+\int_{-1}^{y} \mathrm{~d} z s_{++}(x, z) \mathcal{G}(z, y) \\
& =\delta_{\mathrm{D}}(x-y)-F_{++}(x, y)+\mathrm{H}(y-x) \mathcal{G}(x, y)-V(x, y),
\end{aligned}
$$

where

$V(x, y)=\int_{-1}^{y} \mathrm{~d} z F_{++}(x, z) \mathcal{G}(z, y)$.

Owing to symmetry,

$s_{-+}(x, y)=\sum_{n=1}^{\infty} t_{-n}(x) t_{+n}(y)=s_{+-}(y, x)$,

and

$$
\begin{aligned}
s_{--}(x, y)= & \sum_{n=1}^{\infty} t_{-n}(x) t_{-n}(y) \\
= & s_{++}(x, y)+\mathrm{H}(y-x) \mathcal{G}(x, y) \\
& +\mathrm{H}(x-y) \mathcal{G}(y, x)-V(x, y)-V(y, x)+W(x, y),
\end{aligned}
$$

where the symmetric function $W$ is defined as

$$
\begin{aligned}
W(x, y)= & \int_{-1}^{\min (x, y)} \mathrm{d} z \mathcal{G}(z, x) \mathcal{G}(z, y) \\
& -\int_{-1}^{x} \mathrm{~d} z \int_{-1}^{y} \mathrm{~d} z^{\prime} F_{++}\left(z, z^{\prime}\right) \mathcal{G}(z, x) \mathcal{G}\left(z^{\prime}, y\right)
\end{aligned}
$$

Thus, we find for the $S_{ \pm \pm}(\vartheta, \varphi)$ in turn, using $x=2(\vartheta-\bar{\vartheta}) / \Delta \vartheta$ and $y=2(\varphi-\bar{\vartheta}) / \Delta \vartheta$ :

$$
\begin{aligned}
S_{++}(\vartheta, \varphi)= & \frac{\Delta \vartheta}{2} \delta_{\mathrm{D}}(\vartheta-\varphi)-F_{++}(x, y) \\
S_{+-}(\vartheta, \varphi)= & \frac{\Delta \vartheta}{2} \delta_{\mathrm{D}}(\vartheta-\varphi)-F_{+-}(x, y)+\mathrm{H}(\varphi-\vartheta) \mathcal{G}(x, y) \\
S_{-+}(\vartheta, \varphi)= & S_{+-}(\varphi, \vartheta) \\
S_{--}(\vartheta, \varphi)= & \frac{\Delta \vartheta}{2} \delta_{\mathrm{D}}(\vartheta-\varphi)+\mathrm{H}(\varphi-\vartheta) \mathcal{G}(x, y) \\
& +\mathrm{H}(\vartheta-\varphi) \mathcal{G}(y, x)-F_{--}(x, y)
\end{aligned}
$$

with $F_{+-}(x, y)=F_{++}(x, y)+V(x, y), F_{--}(x, y)=F_{++}(x, y)+$ $V(x, y)+V(y, x)-W(x, y)$. We finally obtain for the pure mode correlation functions

$$
\begin{aligned}
\xi_{+}^{\mathrm{E}, \mathrm{B}}(\vartheta)= & \frac{\xi_{+}(\vartheta)+\mu \xi_{-}(\vartheta)}{2} \\
& -\int_{\vartheta_{\min }}^{\vartheta_{\max }} \frac{\mathrm{d} \varphi \varphi}{\vartheta \Delta \vartheta}\left[\xi_{+}(\varphi) F_{++}\left(\frac{2(\vartheta-\bar{\vartheta})}{\Delta \vartheta}, \frac{2(\varphi-\bar{\vartheta})}{\Delta \vartheta}\right)\right. \\
& \left.+\mu \xi_{-}(\varphi) F_{+-}\left(\frac{2(\vartheta-\bar{\vartheta})}{\Delta \vartheta}, \frac{2(\varphi-\bar{\vartheta})}{\Delta \vartheta}\right)\right] \\
& +\mu \int_{\vartheta}^{\vartheta_{\max }} \frac{\mathrm{d} \varphi \varphi}{\vartheta \Delta \vartheta} \xi_{-}(\varphi) \mathcal{G}\left(\frac{2(\vartheta-\bar{\vartheta})}{\Delta \vartheta}, \frac{2(\varphi-\bar{\vartheta})}{\Delta \vartheta}\right) \\
\xi_{-}^{\mathrm{E}, \mathrm{B}}(\vartheta)= & \frac{\xi_{+}(\vartheta)+\mu \xi_{-}(\vartheta)}{2} \\
& +\int_{\vartheta_{\min }}^{\vartheta^{\vartheta}} \frac{\mathrm{d} \varphi \varphi}{\vartheta \vartheta}\left(\frac{2(\varphi-\bar{\vartheta})}{\Delta \vartheta}, \frac{2(\vartheta-\bar{\vartheta})}{\Delta \vartheta}\right)\left[\xi_{+}(\varphi)+\mu \xi_{-}(\varphi)\right] \\
& +\mu \int_{\vartheta}^{\vartheta_{\max }} \frac{\mathrm{d} \varphi \varphi}{\vartheta \Delta \vartheta} \xi_{-}(\varphi) \mathcal{G}\left(\frac{2(\vartheta-\bar{\vartheta})}{\Delta \vartheta}, \frac{2(\varphi-\bar{\vartheta})}{\Delta \vartheta}\right) \\
& -\int_{\vartheta_{\min }}^{\vartheta_{\max }} \frac{\mathrm{d} \varphi \varphi}{\vartheta \Delta \vartheta}\left[\xi_{+}(\varphi) F_{+-}\left(\frac{2(\varphi-\bar{\vartheta})}{\Delta \vartheta}, \frac{2(\vartheta-\bar{\vartheta})}{\Delta \vartheta}\right)\right. \\
& \left.+\mu \xi_{-}(\varphi) F_{--}\left(\frac{2(\vartheta-\bar{\vartheta})}{\Delta \vartheta}, \frac{2(\varphi-\bar{\vartheta})}{\Delta \vartheta}\right)\right] .
\end{aligned}
$$

Hence, pure mode correlation functions can be obtained from the observed correlation functions over a finite interval. However, we believe that these pure mode correlation functions are of little practical use, since for a quantitative analysis of cosmic shear surveys the COSEBIs contain all relevant information.

\section{References}

Albrecht, A., Bernstein, G., Cahn, R., et al. 2006 [arXiv: astro-ph/0609591] Bartelmann, M., \& Schneider, P. 2001, Phys. Rep., 340, 291

Bassett, B. A., Fantaye, Y., Hlozek, R., \& Kotze, J. 2009 [arXiv: 0906. 0993] Benjamin, J., Heymans, C., Semboloni, E., et al. 2007, MNRAS, 381, 702 Bridle, S., \& King, L. 2007, New J. Phys., 9, 444

Catelan, P., Kamionkowski, M., \& Blandford, R. D. 2001, MNRAS, 320, L7 Crittenden, R. G., Natarajan, P., Pen, U., \& Theuns, T. 2001, ApJ, 559, 552 Crittenden, R. G., Natarajan, P., Pen, U.-L., \& Theuns, T. 2002, ApJ, 568, 20 Efstathiou, G., Bond, J. R., \& White, S. D. M. 1992, MNRAS, 258, 1P Eifler, T., Kilbinger, M., \& Schneider, P. 2008, A\&A, 482, 9 
A\&A 520, A116 (2010)

Eifler, T., Schneider, P., \& Hartlap, J. 2009, A\&A, 502, 721

Eifler, T., Schneider, P., \& Krause, E. 2010, A\&A, 510, A26 Fu, L., \& Kilbinger, M. 2010, MNRAS, 401, 1264

Fu, L., Semboloni, E., Hoekstra, H., et al. 2008, A\&A, 479, 9

Hartlap, J., Simon, P., \& Schneider, P. 2007, A\&A, 464, 399

Hetterscheidt, M., Simon, P., Schirmer, M., et al. 2007, A\&A, 468, 859

Heymans, C., \& Heavens, A. 2003, MNRAS, 339, 711

Hilbert, S., Hartlap, J., White, S. D. M., \& Schneider, P. 2009, A\&A, 499, 31

Hirata, C. M., \& Seljak, U. 2004, Phys. Rev. D, 70, 063526

Hoekstra, H., Yee, H. K. C., \& Gladders, M. D. 2002, ApJ, 577, 595

Jain, B., Seljak, U., \& White, S. 2000, ApJ, 530, 547

Jarvis, M., Bernstein, G. M., Fischer, P., et al. 2003, AJ, 125, 1014

Jarvis, M., Bernstein, G., \& Jain, B. 2004, MNRAS, 352, 338

Jing, Y. P. 2002, MNRAS, 335, L89

Jing, Y. P., Zhang, P., Lin, W. P., Gao, L., \& Springel, V. 2006, ApJ, 640, L119

Joachimi, B., \& Bridle, S. L. 2009, A\&A, accepted [arXiv:0911 .2454]

Joachimi, B., \& Schneider, P. 2008, A\&A, 488, 829

Joachimi, B., \& Schneider, P. 2009, A\&A, 507, 105

Joachimi, B., Schneider, P., \& Eifler, T. 2008, A\&A, 477, 43

Kaiser, N. 1995, ApJ, 439, L1

Kaiser, N. 1998, ApJ, 498, 26
Kilbinger, M., Schneider, P., \& Eifler, T. 2006, A\&A, 457, 15

King, L., \& Schneider, P. 2002, A\&A, 396, 411

King, L. J., \& Schneider, P. 2003, A\&A, 398, 23

Mellier, Y. 1999, ARA\&A, 37, 127

Munshi, D., Valageas, P., van Waerbeke, L., \& Heavens, A. 2008, Phys. Rep., 462,67

Refregier, A. 2003, ARA\&A, 41, 645

Rudd, D. H., Zentner, A. R., \& Kravtsov, A. V. 2008, ApJ, 672, 19

Schneider, P. 1996, MNRAS, 283, 837

Schneider, P., \& Kilbinger, M. 2007, A\&A, 462, 841

Schneider, P., van Waerbeke, L., Jain, B., \& Kruse, G. 1998, MNRAS, 296, 873

Schneider, P., van Waerbeke, L., \& Mellier, Y. 2002, A\&A, 389, 729

Schneider, P., Kilbinger, M., \& Lombardi, M. 2005, A\&A, 431, 9

Schneider, P., Kochanek, C. S., \& Wambsganss, J. 2006, Gravitational Lensing: Strong, Weak and Micro (Berlin: Springer-Verlag)

Schrabback, T., Hartlap, J., Joachimi, B., et al. 2010, A\&A, 516, A63

Smith, R. E., Peacock, J. A., Jenkins, A., et al. 2003, MNRAS, 341, 1311

Tegmark, M., Taylor, A. N., \& Heavens, A. F. 1997, ApJ, 480, 22

Wolfram, S. 1991, Mathematica: a system for doing mathematics by computer, ed. S. Wolfram

Zentner, A. R., Rudd, D. H., \& Hu, W. 2008, Phys. Rev. D, 77, 043507 